\title{
Diferencias regionales en el ingreso laboral y el papel de la educación, informalidad laboral y el sector público. El caso de Ecuador
}

Diego Ontaneda Jimánez*

\section{RESUMEN}

El artículo analiza la importancia de la educación, la informalidad laboral y el trabajo público para explicar las brechas de ingreso laboral entre provincias del Ecuador. Para ello se utilizan microdatos de encuestas laborales de 2017, y se emplean métodos de descomposición basados en regresiones de función de influencia recentrada. Se encuentran marcadas diferencias regionales en términos de ingreso laboral. Los resultados de la descomposición indican que la educación es la variable más importante que explica las diferencias de ingreso laboral entre las provincias. El análisis también muestra que una parte significativa de las disparidades regionales de ingreso laboral responden a la informalidad laboral y al empleo público.

Palabras clave: Ecuador, brechas de ingreso, provincias, educación, informalidad laboral, trabajo público.

Clasificación JEL: C21, J31, J45, J46

\section{ABSTRACT}

Regional Differences in Labour Income and the Role of Education, Labour Informality and the Public Sector. The Case of Ecuador.

The article analyzes the importance of education, labor informality and public work in explaining labor income gaps between provinces of

\footnotetext{
Integrante del Grupo de Investigación en Economía Regional en la Facultad de Ciencias Económicas y Administrativas de la Universidad de Cuenca, Ecuador. Correo electrónico: diego. ontanedaj@ucuenca.edu.ec
} 
Ecuador. For this, microdata of labor surveys of 2017 is used. Besides, the study applies decomposition methods based on regressions of recentered influence functions. It is found that there are great differences in terms of labor income. The results of the decomposition indicate that education is the most important variable explaining the differences in labor income between provinces. The results also show that a significant part of the regional income disparities respond to labor informality and public employment.

Keywords: Ecuador, income gaps, provinces, education, labor informality, public works.

JEL Classification: C21, J31, J45, J46

\section{INTRODUCCIÓN}

Al igual que otros países de América Latina, Ecuador ${ }^{1}$ se ha caracterizado por un alto y persistente nivel de desigualdad de ingresos. Durante los últimos años, el país experimenta una importante expansión económica y transformación política. Entre 2003-2017, la economía ecuatoriana creció en términos reales a una tasa promedio de $3.75 \%$ al año, lo cual se asocia a condiciones externas favorables y una mayor participación del Estado en la economía, a través de la expansión del gasto gubernamental en diferentes áreas como infraestructura y el aumento considerable del gasto social. Diversos estudios han documentado los logros de Ecuador en términos de reducción de desigualdad (Ponce y Vos, 2014; Gachet et al., 2016). Durante este periodo la nación experimentó una reducción de la desigualdad de ingresos de aproximadamente 9 puntos de coeficiente de Gini, ubicándose en el valor de 0.46 en el año 2017.

Se reconoce que la evolución de la disparidad en periodos recientes ha estado estrechamente vinculada al comportamiento del mercado de

\footnotetext{
Con una superficie de 283,500 kilómetros cuadrados, la República de Ecuador está dividida en cuatro regiones naturales (amazónica, costa, sierra e insular), 24 provincias, 221 cantones (municipios) y 1,228 parroquias. La provincia es la división política-administrativa de primer nivel en el país. En el estudio se utiliza indistintamente los términos provincia y región (ver Figura A.1 del anexo
} 
trabajo. En efecto, la desigualdad del ingreso laboral, principal componente del ingreso de los hogares, registró una disminución de 0.11 puntos, y la dispersión salarial ${ }^{2}$ disminuyó en 0.22 puntos (ver Figura A.2 del anexo). Gasparini y Lustig (2011) y Lustig et al. (2012) explican que la reciente disminución de la desigualdad de ingresos en América Latina responde a una combinación de elementos entre los que destaca la caída en la brecha de ingresos entre los trabajadores calificados y poco calificados, lo cual se debe a un amplio conjunto de factores, como la mejora de las condiciones macroeconómicas que fomentaron el empleo, el boom del precio de las materias primas, la expansión de la cobertura en educación durante las últimas dos décadas, así como la presencia de instituciones laborales más fuertes. Los estudios enfocados en Ecuador, por su parte, indican que la reducción de la desigualdad de ingresos durante los últimos años responde a diferentes explicaciones. Ponce y Vos (2014) y Gachet et al. (2016) sugieren que la mejora de la distribución de ingreso responde al incremento de los precios internacionales de las materias primas que permitió la recuperación del país después de la crisis del 1999 y que hizo posible la expansión de programas de transferencia de efectivo por parte del gobierno, el empleo público, el aumento del salario mínimo y los beneficios de la seguridad social.

No obstante, las diferencias en el ingreso laboral entre regiones es un tema que se ha estudiado de forma limitada. Si bien la tendencia nacional en Ecuador es hacia la disminución de la desigualdad, se observan marcadas diferencias en los ingresos laborales al interior del territorio. Por ejemplo, en 2017, el ingreso laboral promedio en Bolívar, la provincia con el menor nivel, fue el 53\% de los ingresos laboralespromedio de Pichincha, la que tiene el nivel más alto, y esta tendencia persiste en el tiempo (Gráfica 2). Diversas investigaciones empíricas dan cuenta de las persistentes disparidades geográficas en el país, reflejadas en una heterogénea geografía económica y social (Muñoz y Pontarollo, 2016; Szeles y Muñoz, 2016; Quintana-Romero et al., 2019). Los estudios muestran una economía regional caracterizada por una gran heterogeneidad y altamente polarizada. De 24 provincias ecuatorianas, tres (Pichincha, Guayas y Azuay) generalmente tienen una mejor posición

2 La dispersión salarial se mide como la varianza del logaritmo natural del salario real por hora. 
relativa en comparación con las demás. Las diferentes investigaciones también muestran la existencia de un lento proceso de convergencia del ingreso per cápita y productividad regional, con un nivel elevado de segregación espacial (Muñoz y Pontarollo, 2016; Quintana-Romero et al., 2019; Pontarollo et al., 2019).

Si bien diversas investigaciones dan cuenta de las marcadas inequidades regionales en Ecuador, pocos estudios empíricos identifican los factores que explican estas diferencias. Los análisis que toman en cuenta la dimensión regional son indispensables, porque incluso en presencia de una disminución de la desigualdad de ingresos a nivel global, pueden persistir marcadas disparidades entre regiones. Estas asimetrías territoriales pueden inhibir el crecimiento nacional y afectar el desarrollo futuro de todo el país (Muñoz y Pontarollo, 2016). Una gran cantidad de literatura ha demostrado que las distribuciones de ingreso menos iguales se asocian con un menor crecimiento y pueden producir grandes costos sociales (Dabla-Norris et al., 2015; Cingano, 2014). Se considera que la elevada desigualdad puede reducir el crecimiento económico al privar a los hogares de menores ganancias de la capacidad de acumular capital físico y humano; la concentración elevada de ingresos puede reducir la demanda, y la desigualdad extrema puede representar severos riesgos de estabilidad social.

Tradicionalmente se considera al capital humano como uno de los principales determinantes del ingreso laboral; la tasa salarial del trabajador depende de forma positiva del número de años de educación y de los años de experiencia en el mercado laboral (Becker, 1964; Mincer, 1974). No obstante, una de las principales características de este mercado es un empleo informal considerable. De acuerdo con información del Instituto Nacional de Estadísticas y Censos de Ecuador, la tasa de empleo informal, entendida como la falta de cobertura de seguridad social $^{3}$ durante 2017 representaba aproximadamente $55 \%$, y se observa que la incidencia de la informalidad laboral en Ecuador no está igualmente distribuida entre el territorio del país (Anexo A.3). En 2017, en Bolívar esta tasa superaba en aproximadamente 23 puntos porcentuales a Pichincha, la de menor nivel (Tabla 1). Se determina que la informa- 
lidad laboral se presenta principalmente en las provincias de la región natural amazónica, en donde representa en promedio 59\% del empleo, mientras que en las tres provincias más económicamente dinámicas del país, se ubica en alrededor de 50\% (ver Tabla A.1 del anexo). Esta situación puede contribuir a explicar las diferencias en los niveles de ingreso laboral entre las regiones dado que, como diversos estudios han demostrado, si se mantienen constantes otras características, los trabajadores informales registran una reducción significativa de sus tasas salariales en comparación con los trabajadores formales.

Por otro lado, el sector público ha jugado un rol importante en la economía ecuatoriana. En 2017, el porcentaje de trabajadores públicos llegó a representar $9.3 \%$ del empleo total. El número de empleados de este sector ha incrementado de forma significativa durante los últimos años, pasando de 460,182 trabajadores (7.6\% del empleo) en 2007 a 718,796 (9.3\% del empleo) en 2017. De igual forma, se observan marcadas discrepancias regionales en la importancia relativa del empleo público (ver Figura A.3 del anexo). Esta situación puede afectar los niveles de ingreso regionales dado que la evidencia ha señalado la existencia de una prima salarial a favor del empleo público.

A pesar de estas características claras del mercado laboral ecuatoriano, su importancia no ha sido considerada al analizar las diferencias regionales en el nivel de ingreso. Así, el presente trabajo estudia la importancia de las discrepancias en el nivel de educación, informalidad laboral y empleo público para explicar las brechas salariales regionales en Ecuador. En este sentido, se sostiene que las diferencias en el ingreso laboral entre las regiones ecuatorianas se pueden explicar por disparidades asociadas con la educación, trabajo informal y empleo público. El análisis de los factores que explican las brechas regionales de ingreso es un tema poco abordado en investigaciones en Ecuador.

El estudio utiliza métodos de descomposición basados en regresiones de función de influencia recentrada (Firpo et al., 2009; Fortin et al., 2011) para estimar el impacto cuantitativo de cada uno de los factores sobre la brecha de ingresos laboral entre las regiones ecuatorianas. Para cada variable explicativa, se determina qué se puede atribuir a las diferencias observables en las características de los trabajadores (efecto composición), y lo que se debe a las diferencias en las primas asociadas con esas características (el efecto precio). Para ello, se aplica la descomposición estándar de Oaxaca-Blinder en la media y la 
descomposición para la regresión cuantil incondicional, lo que permite aislar la contribución de cada factor sobre la brecha regional de ingresos laborales a lo largo de la distribución. La principal ventaja del método propuesto es que permite identificar la contribución de una covariable o grupos de covariables en la explicación de las diferencias regionales de ingreso en la media y otras estadísticas distribucionales, como cuantiles, lo que hace posible un mejor entendimiento de las causas de las diferencias salariales regionales.

El presente documento se estructura de la siguiente forma, además de la introducción y las conclusiones: en la sección uno se hace una breve revisión de literatura sobre los factores que explican las diferencias de ingreso regionales y familiariza al lector con la literatura relacionada con la informalidad laboral y empleo público. En las secciones dos y tres se describe la metodología utilizada y se realiza un análisis descriptivo preliminar. La sección cuatro presenta los resultados de la investigación.

\section{REVISIÓN DE LITERATURA}

La distribución del capital humano es uno de los principales determinantes de la distribución salarial. La teoría del capital humano explica que los ingresos laborales pueden responder a distintos factores, entre estos se encuentra la productividad, la educación y la experiencia. Becker (1964) establece una relación entre educación e ingresos laborales, al señalar que las características inherentes de los individuos, que están relacionadas con sus habilidades, capacidades y conocimientos, son fuentes generadoras de ganancias. Por su parte, Mincer (1974) señala que las diferencias salariales entre sujetos se producen principalmente por la acumulación de capital humano, a través de la inversión en educación, salud y capacitación. Demuestra que el modelo de capital humano genera una función en la que la tasa salarial del trabajador depende de forma positiva del número de años de escolaridad y de la cantidad de años de experiencia en el mercado laboral. Así, el modelo de capital humano implica que la distribución de ingresos laborales está determinada principalmente por el nivel y la distribución de la educación.

Una característica distintiva de los mercados laborales en países latinoamericanos es la informalidad, que generalmente representa una 
proporción significativa del trabajo (Gasparini y Tornarolli, 2009 y Maurizio, 2014). A pesar de ello, los estudios previos sobre las disparidades salariales en Ecuador han ignorado la relevancia del trabajo informal y cómo su incidencia puede explicar las brechas de ingreso laboral entre las regiones.

La informalidad laboral no tiene una definición única; se trata de un concepto que ha evolucionado con el tiempo (Perry et al., 2007). De acuerdo con Maloney (2004) y Perry et al. (2007), existen principalmente dos perspectivas que buscan explicar su existencia. Por un lado, el sector informal surge como resultado de la poca capacidad del sector formal para absorber mano de obra, por lo que los individuos, antes de quedarse desempleados, prefieren ubicarse en la informalidad. Por otro lado, Maloney (2004) y Perry et al.(2007) señalan que ciertos trabajadores, particularmente los autoempleados y microempresarios, optan voluntariamente por salir del sector formal e incorporarse a la informalidad laboral dependiendo del valor que asignen a los beneficios de la formalidad y la capacidad de fiscalización del Estado.

El consenso sugiere que el sector informal es una combinación de trabajadores segmentados y autoseleccionados (Perry et al., 2007). Cabe mencionar que actualmente no existe consenso sobre la magnitud de las brechas salariales entre trabajadores formales e informales. La evidencia ha proporcionado resultados mixtos. En un estudio para México, Maloney (1999), al considerar a los autoempleados como trabajadores informales, encuentra que los ingresos laborales promedio en el sector informal son mayores que los salarios de los trabajadores del sector formal. Por otro lado, al analizar las brechas salariales asociadas a este sector en un conjunto de países latinoamericanos, Gasparini y Tornarolli (2009) y Maurizio (2013) determinan que los trabajadores informales registran una reducción significativa de sus ingresos laborales respecto a los formales. La literatura que analiza el impacto de la informalidad laboral sobre la desigualdad del ingreso es escasa; el consenso define que una mayor informalidad laboral se asocia con un incremento de la desigualdad salarial (Atanassio y Binelli, 2010; Binelli, 2016; Amarante et al., 2016; Alejo y Parada, 2017; Ontaneda, 2019).

A nivel internacional, diversos estudios han analizado las diferencias salariales entre trabajadores del sector público y privado. La mayoría indica que existe un diferencial salarial a favor de los trabajadores del 
sector público, aun cuando se controlan por características personales asociadas a la productividad. La evidencia muestra que este diferencial salarial se presenta tanto en la media (Amarante, 2001; Panizza y Qiang, 2005; Giordano Giordano et al., 2011; De Castro et al., 2013), como a lo largo de la distribución salarial (Mueller, 1998; Lucifora y Meurs, 2006; Su et al., 2019). De la misma manera, se señalan marcadas diferencias regionales en la brecha salarial entre el sector público y privado (Dell'Aringa et al., 2007). En el caso de Ecuador, diversos estudios han analizado los diferenciales salariales público-privado (Carrillo, 2004; Guerrero, 2013; Maldonado et al., 2018) y presentan evidencia de una prima salarial a favor de los trabajadores del sector público.

Desde el punto de vista teórico, las diferencias de ingreso laboral entre regiones se pueden explicar de varias formas. Al respecto, Combes et al. (2008) se basan en la composición de habilidades de la fuerza laboral, en donde los individuos con mejores características tienden a aglomerarse en mercados laborales más grandes, densos y calificados. Adicionalmente, las diferencias salariales espaciales pueden ocurrir como resultado de las características geográficas de los territorios, así como por interacciones entre trabajadores o empresas que producen ventajas en la productividad. Por su parte, Glaeser et al. (1992) enfatizan la importancia de los derrames de conocimiento, por lo cual la aglomeración espacial de individuos, ocupaciones e industrias permite la fácil transmisión de ideas entre personas y empresas, lo que incrementa la innovación y la productividad.

De igual forma, la literatura resalta la importancia de las economías de escala y los costos de transporte para crear externalidades asociadas a los vínculos de demanda y oferta, los cuales determinan la divergencia regional. Estos modelos explican las diferencias en las tasas salariales entre regiones como resultado del efecto del tamaño del mercado interno, el nivel de precios y el grado de competencia en el mercado laboral local (Krugman, 1991).

Investigaciones recientes han enriquecido nuestro conocimiento sobre la heterogeneidad regional del ingreso laboral. Por ejemplo, Kosfeld y Eckey (2010), basados en el marco teórico de la Nueva Geografía Económica (NEG), sostienen que las empresas ubicadas en regiones con buen acceso a grandes mercados experimentan mayor demanda de sus productos y son capaces de pagar salarios más altos debido al ahorro en costos de transporte y al incremento de los retornos 
a escala. Por su parte, Zhou (2017) integra los costos urbanos en los modelos de la NEG y encuentra que la interacción de éstos con los costos de transporte de los bienes manufactureros determina los salarios urbanos y la distribución económica. Sus resultados muestran que los diferenciales salariales y los costos de transporte mantienen un patrón en forma de campana y que los diferenciales salariales urbanos y los costos de commuting mantienen una relación monotónica.

Diversos estudios han analizado las brechas salariales regionales desde el punto de vista empírico. En términos generales, la estrategia metodológica instrumentada consiste en estimar una ecuación salarial para, posteriormente, llevar a cabo una descomposición de la brecha de ingresos entre regiones. Éste consiste en determinar en qué grado las diferencias salariales observadas entre trabajadores se pueden atribuir a discrepancias, respecto a características de los trabajadores, y a variaciones, en cuanto al retorno a estas características. El análisis se puede realizar tanto en la media como en diferentes puntos de la distribución, lo que permite un mejor entendimiento de las causas de las brechas regionales de salarios. Por ejemplo, Herrera-Idárraga et al. (2016) analizan el caso de Colombia. Los autores estiman los retornos a la educación y la penalidad del trabajo informal de las regiones colombianas; posteriormente se descomponen las brechas salariales en la contribución de la distribución regional de las características y la estructura salarial. Los resultados indican que una importante parte de las disparidades salariales regionales se explican por desiguladades en educación y trabajo informal. Otro estudio pertinente es el de Pereira y Galego (2014), quienes analizan el caso de Portugal; determinan que las brechas salariales regionales se demuestran tanto por diferencias de características como de los retornos. Las particularidades más importantes que describen las brechas salariales son el porcentaje de empresas grandes, el nivel de educación y la ocupación. Respecto a las diferencias en los retornos, éstas son explicadas principalmente por variaciones regionales en el retorno a la educación. Galego y Pereira (2014), al descomponer las diferencias regionales de ingreso salarial en Portugal, encuentran que la educación, la ocupación y el tamaño de las empresas esclarecen el efecto composición, mientras que las diferencias en los retornos a la experiencia y permanencia en el trabajo determinan el efecto estructura salarial. El análisis también revela que la 
importancia de estas variables no es homogénea a lo largo de la distribución salarial.

De igual forma, Motellón et al. (2011) analizan el efecto del capital humano en las diferencias salariales regionales en España. Los resultados muestran que las dotaciones de capital humano y los retornos obtenidos de los trabajadores varían significativamente entre regiones. Así, las desemejanzas en las dotaciones y en los retornos al capital humano representan la mayor parte de las brechas salariales regionales. En un estudio similar, García y Molina (2002) estudian las diferencias salariales entre las regiones españolas. En este caso, los resultados indican que el nivel de educación tiene un efecto limitado en las diferencias salariales totales. Las variables que representan las mayores diferencias regionales, tanto en características como en remuneración, son la antigüedad en el puesto de trabajo, el manejo de un segundo idioma y la brecha salarial de género.

En el caso de Gran Bretaña, Dickey (2007) analiza la evolución de la desigualdad salarial dentro de las regiones y los factores que han determinado su incremento entre 1976-1995. De acuerdo con los resultados, el aumento de los retornos a la ocupación y edad han contribuido a la ampliación de la desigualdad regional. Asimismo, tanto la sindicalización como la reducción de la brecha salarial de género tienen un efecto igualador sobre la desigualdad dentro de las regiones.

El análisis de las brechas salariales regionales no se ha llevado a cabo exclusivamente con metodologías de descomposición. Groot et al. (2014) analizan las fuentes de diferencias salariales regionales de ingreso en Países Bajos considerando la parte de los salarios no explicada por las características del trabajador en una ecuación minceriana. Los resultados indican que una proporción importante de las diferencias salariales regionales responde a varios tipos de externalidades, como el tamaño del mercado laboral regional, los derramamientos de conocimiento intrasectoriales (externalidades Marshall-Arrow Romer), la competencia (externalidades Porter) y la diversidad (externalidades Jacobs).

En resumen, los estudios determinan que las diferencias salariales regionales se deben tanto a desemejanzas espaciales en características de los trabajadores, como a discrepancias en los retornos. En la mayoría de los estudios se identifica la educación como la variable más importante para explicar las disparidades salariales regionales. No 
obstante, también se precisa que existe poca evidencia disponible sobre la magnitud y el origen de las diferencias de ingreso regionales en los países latinoamericanos.

La mayoría de los análisis de brechas salariales entre grupos de trabajadores utilizan el método de Oaxaca-Blinder, que permite descomponer las diferencias en la media de la distribución salarial en efecto composición y efecto precio. No obstante, los estudios que aplican este enfoque para analizar las diferencias regionales de salarios (García y Molina, 2002) no consideran que los factores importantes en la media de la distribución pueden no tener la misma relevancia en la parte baja o en la parte superior de la distribución. Actualmente, se dispone de varias metodologías que permiten analizar las diferencias salariales a lo largo de la distribución, entre ellos el método de Machado y Mata (2005), que extiende la descomposición de Oaxaca-Blinder a regresiones cuantílicas, y el de DiNardo et al. (1996), que aplica un procedimiento de reponderación para calcular el efecto composición correspondiente a una variable binaria. Éstos han sido utilizados para analizar las diferencias salariales regionales por Pereira y Galego (2014) y por Motellon et al. (2011). No obstante, el primero no permite descomponer las diferencias salariales en la contribución de cada covariable, mientras que los resultados del enfoque de DiNardo et al. (1996) están condicionados al orden en el que se incluyen las covariables en la descomposición. ${ }^{4}$

El estudio utiliza un método de descomposición más reciente, que supera estas limitaciones, propuesto por Firpo et al. (2009) y Fortin et al. (2011). Con él se analizaron previamente las diferencias regionales de salarios por Galego y Pereira (2014) y Herrera-Idárraga et al. (2016). La principal ventaja de este enfoque frente a otros es que permite obtener una descomposición detallada de la contribución de cada covariable para explicar el efecto-composición y el efecto-precio en la media y otras estadísticas distribucionales, como cuantiles, lo que hace posible determinar la importancia de cada factor para explicar las diferencias salariales a lo largo de la distribución. De esta forma, permite obtener un análisis más detallado de las causas de las diferencias salariales entre

4 Para una detallada descripción de las ventajas y desventajas de los métodos de descomposición propuestos en la literatura véase Fortin et al. (2011). 
regiones y establecer medidas de política más efectivas orientadas a factores específicos y rangos concretos de la distribución.

\section{Metodología}

El análisis inicia con la siguiente ecuación salarial:

$$
Y_{g}=X_{g} \beta_{g}+u_{g}, \quad g=j, k
$$

Donde $Y$ representa el logaritmo del ingreso laboral por hora, y el subíndice $g$, la región. $X$ denota un vector de covariables que determinan el ingreso laboral de los individuos, incluyendo: i) capital humano; ii) características demográficas; iii) condición laboral; iv) distribución sectorial. $\beta_{g}$ es el vector de retornos de la región $g$.

Después de estimar las ecuaciones salariales por el método de mínimos cuadrados ordinarios (MCO), se aplica el enfoque propuesto por Oaxaca (1973) y Blinder (1973) para descomponer la diferencia en la media de los ingresos laborales entre dos regiones, $\mathrm{j} \mathrm{y} \mathrm{k}$.

$$
\begin{gathered}
\hat{\Delta}_{O}^{u}=\bar{Y}_{j}-\bar{Y}_{k}=\bar{X}_{j} \hat{\beta}_{j}-\bar{X}_{j} \hat{\beta}_{k}+\bar{X}_{j} \hat{\beta}_{k}-\bar{X}_{k} \hat{\beta}_{k} \\
\hat{\Delta}_{O}^{u}=\bar{X}_{j}\left(\hat{\beta}_{j}-\hat{\beta}_{k}\right)+\left(\bar{X}_{j}-\bar{X}_{k}\right) \hat{\beta}_{k} \\
\hat{\Delta}_{O}^{u}=\hat{\Delta}_{P}^{u}+\hat{\Delta}_{C}^{u}
\end{gathered}
$$


Donde la barra superior representa el valor de la media muestral y $\hat{\beta}$, representa los coeficientes estimados por el método de MCO de la ecuación 1. El término de la izquierda indica la diferencia entre los ingresos laborales promedio entre las regiones $\mathrm{j}$ y $\mathrm{k}$. El primer término del lado derecho refleja la parte de esta diferencia debido a las diferentes remuneraciones de las características (efecto precio), y el segundo término indica la parte de la diferencia debido a las diferentes características de los trabajadores entre las regiones (efecto composición).

Esta descomposición se pueden utilizar para analizar las diferencias en los ingresos laborales en la media de los ingresos y no sobre la distribución de ingresos en su conjunto, lo que puede ocultar aspectos importantes de sobre cómo las variables explicativas afectan el ingreso laboral. En este sentido, es necesario conocer el efecto de las covariables en diferentes puntos de la distribución de los ingresos laborales.

Para determinar el impacto de cada variable explicativa a lo largo de la distribución del ingreso laboral, el estudio utiliza el enfoque propuesto por Firpo et al. (2009) sugerido por Fortin et al. (2011), basado en la función de influencia (IF) y la función de influencia recentrada (RIF). La IF correspondiente a un ingreso observado Y, para el $\tau$ ésimo cuantil $q_{\tau}$, está definido por la siguiente expresión:

$$
I F\left(Y ; q_{\tau}\right)=\frac{\tau-\mathbb{1}\left\{Y \leq q_{\tau}\right\}}{f_{Y}\left(q_{\tau}\right)}
$$

Donde $f_{Y}\left(q_{\tau}\right)$ es la función de densidad de probabilidad de $Y$ evaluada en $q_{\tau}$ : $1\left\{Y<q_{\tau}\right\}$ es una variable indicadora que señala si $Y$ es menor que $q_{\tau}$. La RIF se define como $\operatorname{RIF}\left(Y ; q_{\tau}\right)=q_{\tau}+\operatorname{IF}\left(Y ; q_{\tau}\right)$. Asumiendo que la función de influencia recentrada es lineal en las covariables $X$, se pueden estimar los parámetros a través de una regresión de MCO:

$$
E\left[R I F\left(Y ; q_{\tau}\right) \mid X\right]=X \hat{\gamma}_{\tau}
$$


Donde $\hat{\gamma}_{\tau}$ representan los parámetros estimados por medio de MCO, y miden los efectos marginales aproximados de las variables explicativas sobre el cuantil $q_{\tau}$ de los ingresos laborales de los trabajadores. Al respecto, Firpo et al. (2009) explican que la estimación de la RIF consiste de dos pasos. Primero se calcula la RIF del tésimo cuantil de $Y$ y, segundo, se lleva a cabo una regresión MCO de la $\operatorname{RIF}\left(Y ; q_{\tau}\right)$ sobre las covariables $X$.

Después de estimar los coeficientes de la regresión cuantil incondicional para cada región, j y k, se puede escribir el equivalente de la descomposición Oaxaca-Blinder para cualquier cuantil incondicional de la siguiente forma:

$$
\begin{gathered}
\hat{\Delta}_{O}^{\tau}=\bar{X}_{j}\left(\hat{\gamma}_{j, \tau}-\hat{\gamma}_{k, \tau}\right)+\left(\bar{X}_{j}-\bar{X}_{k}\right) \hat{\gamma}_{k, \tau} \\
\hat{\Delta}_{O}^{\tau}=\hat{\Delta}_{P}^{\tau}+\hat{\Delta}_{C}^{\tau}
\end{gathered}
$$

La descomposición detallada permite calcular la contribución de cada variable explicativa $(l=1 \ldots L)$ a la estructura de precios y características.

$$
\begin{aligned}
& \hat{\Delta}_{P}^{\tau}=\sum_{l=1}^{L} \bar{X}_{j l}\left(\hat{\gamma}_{j l, \tau}-\hat{\gamma}_{k l, \tau}\right) \\
& \hat{\Delta}_{C}^{\tau}=\sum_{l=1}^{L}\left(\bar{X}_{j l}-\bar{X}_{k l}\right) \hat{\gamma}_{k l, \tau}
\end{aligned}
$$

Específicamente, el estudio analiza la contribución del nivel de educación, la condición de informalidad y el empleo público.

\section{DESCRIPCIÓN DE LOS DATOS Y ANÁLISIS PRELIMINAR}

La principal fuente de información corresponde la Encuesta Nacional de Empleo y Desempleo del Ecuador (Enemdu), la base de datos nacional más importante para la medición, seguimiento y caracterización del mercado laboral, realizada por el Instituto Nacional de Estadísticas y Censos. La encuesta está dirigida a los hogares y sus habitantes, 
y recopila información sobre las características individuales de cada miembro del hogar, condiciones laborales, salarios de los empleados asalariados, ingresos de los trabajadores por cuenta propia y las horas trabajadas para todas las personas empleadas. Igualmente, presenta información para tres fuentes de ingresos laborales: trabajo principal, trabajo secundario y otros trabajos. La variable de ingresos utilizada en este estudio se refiere al ingreso laboral por hora y se expresan en términos reales utilizando el índice de precios al consumidor, año base 2014, y se calcula a partir del ingreso laboral mensual por la ocupación principal informado por los trabajadores, dividido por la cantidad de horas trabajadas habituales. Se considera a los individuos con edades entre los 15-70 años. La muestra incluye empleados del sector público, asalariados privados, trabajadores y empleados domésticos, autoempleados y patrones. Aquellos que trabajan sin remuneración no fueron considerados. Las encuestas corresponden al mes de diciembre. Los pesos muestrales proporcionados por la encuesta son utilizados en todos los análisis.

La informalidad laboral se determina siguiendo una definición legal, mientras que un trabajador informal es aquel "cuya relación laboral no está sujeta a la legislación laboral y las normas tributarias, y no tiene acceso a la protección social ni a ciertos beneficios laborales" (Hussmanns, 2004: 7). Específicamente, trabajador informal es aquel que no se encuentra cubierto por el sistema de seguridad social. Se utiliza esta definición porque se sostiene que la medición basada en la cobertura de la seguridad social ofrece una mejor imagen de la proporción de trabajadores informales en el mercado laboral ecuatoriano. ${ }^{5}$

El periodo de estudio corresponde al 2017, año más reciente del que se dispone de información. Posteriormente, los datos son agregados a nivel de provincia. Actualmente, Ecuador está conformado por 24, pero se decidió no considerar Galápagos debido a que está sujeta a un

\footnotetext{
En Ecuador, la afiliación a la seguridad social es obligatoria para todos los trabajadores, por lo tanto, si el alguno no está afiliado al sistema de seguridad social, es probable que tampoco reciba los beneficios obligatorios de ley. De esta forma, al agrupar a los trabajadores informales según esta definición, se puede determinar la proporción de trabajadores cuya relación laboral cumple con la legislación laboral. Esta medida ha sido empleada en la literatura, por ejemplo por Maloney (2004), por Gasparini y Tornarolli (2009) y, en el caso de Ecuador, por Canelas (2014).
} 
régimen especial que dificulta su comparación con respecto al territorio continental. En este sentido, el análisis cubre todas las provincias de Ecuador continental. Se descartan las observaciones con los ingresos por arriba y por debajo del percentil superior e inferior

La Figura 1 presenta las funciones de densidad para la distribución del logaritmo del ingreso laboral por hora; por simplicidad se han clasificado a las provincias de acuerdo a la región natural. Las funciones de densidad están basadas en un kernel Gaussiano con ancho de banda óptimo dado por la regla sugerida por Silverman (1986). Las principales diferencias en la forma de estas distribuciones parecen estar entre la región natural Costa y las otras regiones. La densidad de la región Costa se encuentra a la izquierda de las otras regiones, y la distribución para esta región sugiere una menor dispersión salarial. En contraste, las distribuciones de las otras regiones naturales tienen colas inferiores más amplias y muestran un área de probabilidad más alta en la cola superior y en todo el lado derecho, lo que indica una mayor dispersión del ingreso laboral. En general, esto sugiere que los salarios promedio son más altos en la región Sierra y que las principales diferencias salariales son entre la Costa y todas las demás regiones. Se observa un comportamiento bimodal, con una pequeña acumulación de probabilidad a la derecha de la media. Este comportamiento se puede explicar por los ingresos laborales de los trabajadores del sector público que, como se muestra más adelante, representan aproximadamente $15 \%$ del empleo total y registran ingresos laborales mayores al del resto de trabajadores. El análisis también señala que las brechas de ingreso varían considerablemente a lo largo de la distribución del ingreso laboral. 


\section{FIGURA 1}

ESTIMACIONES SEGÚN REGIÓN NATURAL DE LA DENSIDAD DEL INGRESO LABORAL REAL POR HORA

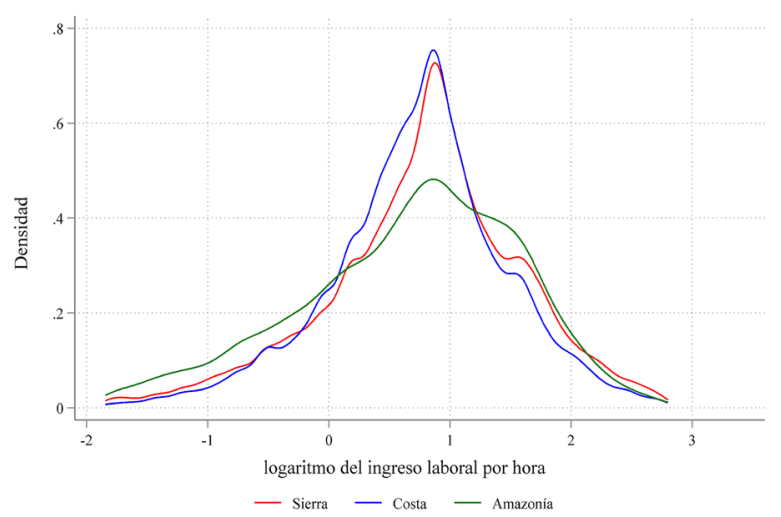

Fuente: elaborado con base en Enemdu.

La Figura 2 presenta el ingreso laboral por hora promedio a nivel provincial. Existen marcadas diferencias entre provincias en términos de ingresos laborales. Por ejemplo, en Pichincha, en el 2017, el ingreso laboral promedio era de 3.46 USD por hora, mientras que en Bolívar, tan sólo 2.40 USD por hora.

FIGURA 2

INGRESO LABORAL POR HORA SEGÚN PROVINCIAS

Ingreso laboral promedio

ㅁ $2.36-2.48$

ㄴ.48 - 2.64

ㅁ $2.64-2.75$

ㅁ $2.75-2.89$

— $2.89-3.68$

Fuente: elaborado con base en Enemdu. 
Para explicar las diferencias regionales en el ingreso laboral, en la Figura A.3 del anexo se presentan estadísticas descriptivas para las principales variables utilizadas en el análisis. Se deduce que existen diferencias importantes en la distribución del capital humano en todo el país; mientras que en Pichincha la escolaridad del empleo fue de 12.24 años, en Los Ríos fue de 9.80 años. Estas diferencias también son evidentes en términos de incidencia de informalidad. Por ejemplo, en Santa Elena, donde hay mayor incidencia de informalidad laboral, la tasa fue de $63 \%$, mientras que en Pichincha, la de menor incidencia, fue de $40 \%$. En cuanto al trabajo del sector público, se observa que aproximadamente $12 \%$ del empleo corresponde al sector público. Por su parte, las provincias de Morona Santiago, Pastaza y Napo presentan la mayor proporción de empleo público respecto al empleo total provincial, mientras que El Oro, Guayas y Los Ríos, el menor porcentaje.

TABLA 1

PROMEDIOS DE MUESTRA: VARIABLES SELECCIONADAS

\begin{tabular}{ccccccc}
\hline & Escolaridad & Edad & Hombre & Mujer & $\begin{array}{c}\text { Trabajo } \\
\text { informal }\end{array}$ & $\begin{array}{c}\text { Trabajo } \\
\text { público }\end{array}$ \\
Nacional & 11.14 & 39.28 & 0.64 & 0.36 & 0.53 & 0.12 \\
Azuay & 11.31 & 38.54 & 0.58 & 0.42 & 0.52 & 0.10 \\
\hline Bolívar & 10.48 & 40.43 & 0.65 & 0.35 & 0.63 & 0.17 \\
Cañar & 9.85 & 39.79 & 0.61 & 0.39 & 0.56 & 0.12 \\
\hline Carchi & 10.32 & 40.23 & 0.63 & 0.37 & 0.61 & 0.14 \\
Chimborazo & 10.35 & 40.30 & 0.60 & 0.40 & 0.56 & 0.15 \\
\hline Cotopaxi & 10.10 & 39.27 & 0.60 & 0.40 & 0.55 & 0.15 \\
\hline El Oro & 11.03 & 39.50 & 0.64 & 0.36 & 0.62 & 0.09 \\
\hline Esmeraldas & 10.68 & 40.42 & 0.69 & 0.31 & 0.54 & 0.19 \\
\hline Luavas & 11.53 & 39.06 & 0.65 & 0.35 & 0.56 & 0.08 \\
\hline Imbabura & 11.14 & 39.50 & 0.60 & 0.40 & 0.53 & 0.16 \\
\hline Loja & 11.66 & 39.57 & 0.64 & 0.36 & 0.49 & 0.19 \\
\hline
\end{tabular}




\begin{tabular}{|c|c|c|c|c|c|c|}
\hline & Escolaridad & Edad & Hombre & Mujer & $\begin{array}{l}\text { Trabajo } \\
\text { informal }\end{array}$ & $\begin{array}{l}\text { Trabajo } \\
\text { público }\end{array}$ \\
\hline Morona Santiago & 11.69 & 39.05 & 0.62 & 0.38 & 0.57 & 0.28 \\
\hline Napo & 11.29 & 39.79 & 0.65 & 0.35 & 0.60 & 0.23 \\
\hline Orellana & 10.59 & 38.10 & 0.71 & 0.29 & 0.59 & 0.15 \\
\hline Pastaza & 11.34 & 38.30 & 0.66 & 0.34 & 0.56 & 0.24 \\
\hline Pichincha & 12.24 & 38.82 & 0.60 & 0.40 & 0.40 & 0.12 \\
\hline Santa Elena & 10.31 & 39.61 & 0.72 & 0.28 & 0.63 & 0.11 \\
\hline $\begin{array}{l}\text { Santo Domingo de } \\
\text { los Tsáchilas }\end{array}$ & 10.81 & 39.13 & 0.64 & 0.36 & 0.61 & 0.10 \\
\hline Sucumbíos & 11.05 & 39.13 & 0.71 & 0.29 & 0.54 & 0.22 \\
\hline Tungurahua & 11.13 & 39.70 & 0.59 & 0.41 & 0.59 & 0.12 \\
\hline Zamora Chinchipe & 11.00 & 39.90 & 0.66 & 0.34 & 0.51 & 0.22 \\
\hline
\end{tabular}

Fuente: elaborado con base en Enemdu.

Nota: Se presentan proporciones, excepto cuando se indica en los paréntesis.

Como se puede ver en la Tabla A.1 del anexo, existen diferencias entre regiones en términos de estructura industrial. Por ejemplo, en Guayas, las industrias más importantes son comercio y servicios como intermediación financiera, administración pública y enseñanza, mientras que en Bolívar, la agricultura, ganadería, caza y silvicultura son cruciales. De este modo, en todas las regresiones se incluyen controles sectoriales.

\section{RESULTADOS Y DISCUSIÓN}

Primero, se analizan los coeficientes estimados para las ecuaciones salariales regionales. El análisis se basa en ecuaciones salariales mincerianas estimadas para cada región a través de $\mathrm{MCO}$ y regresiones cuantílicas incondicionales. Se consideran variables explicativas: i) capital humano; ii) características demográficas; iii) condición laboral; iv) sector de actividad económica.

Las estimaciones por MCO de las ecuaciones salariales regionales se presentan en la Tabla $2 \mathrm{a}$ y $2 \mathrm{~b}$. Todos los coeficientes estimados son significativos y presentan los efectos esperados. Tomando como referencia el análisis nacional, los resultados sugieren que un año adicional de educación incrementa el ingreso laboral en aproximadamente 4\% después de controlar sus características personales, condición laboral, 
trabajo en el sector público y sector de actividad económica. Se determina que un año adicional de edad incrementa el salario en $0.574 \% .{ }^{6}$ Cabe señalar que en promedio un trabajador del género masculino recibe una tasa salarial mayor en $20.6 \%$ respecto al género femenino. Los resultados señalan que la brecha salarial relacionada con la condición de informalidad laboral es negativa y estadísticamente significativa. Esto implica que, si se mantienen constantes otras características, los trabajadores informales registran una reducción significativa de sus tasas salariales en comparación con los trabajadores formales. Asimismo, se determina que un trabajador del sector público obtiene una tasa salarial superior (43.3\%) en comparación a un trabajador del sector privado con características similares.

Respecto a las estimaciones de coeficientes entre regiones, existen diferencias importantes. Por ejemplo, los retornos a la educación son los más altos para las provincias de Azuay y Loja; mientras que Bolívar y Zamora Chinchipe muestran los más bajos. Por su parte, Azuay y Loja también tienen elevados valores para coeficientes de edad. Igualmente, la brecha salarial entre hombres y mujeres es, en general, mayor para Zamora Chinchipe, Azuay y Santo Domingo de los Tsáchilas que para otras provincias. La penalidad salarial de los trabajadores informales varía considerablemente entre regiones. En la provincia de Sucumbíos la magnitud de la brecha salarial es de $48.7 \%$, mientras que en Chimborazo es de $21 \%$. En este sentido, se determina que las provincias ecuatorianas no sólo difieren en la incidencia de la informalidad laboral, sino también en términos de las brechas salariales entre trabajadores informales y formales. De igual forma, se observan marcadas diferencias regionales en los ingresos laborales de los trabajadores del sector público. La provincia de Chimborazo tiene la mayor prima salarial; un trabajador del sector público gana $87.9 \%$ más que uno del sector privado en esa región, mientras que en Pichincha esta tasa salarial es de 32.5 por ciento.

6 Se calcula como $0.032+2(-0.000361)(36.37)$. 


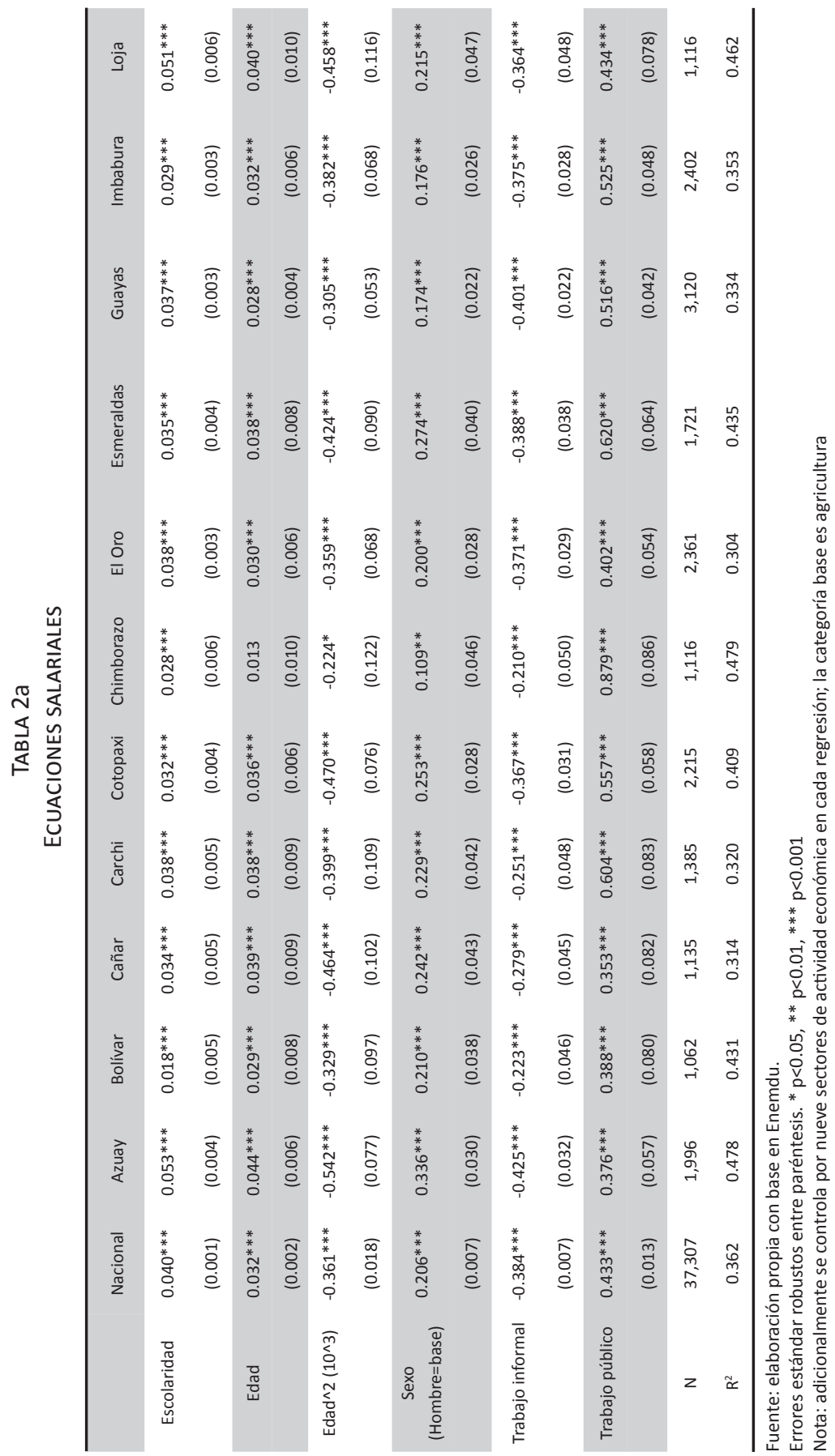


Paradigma económico 7 Año 12 Núm. 1

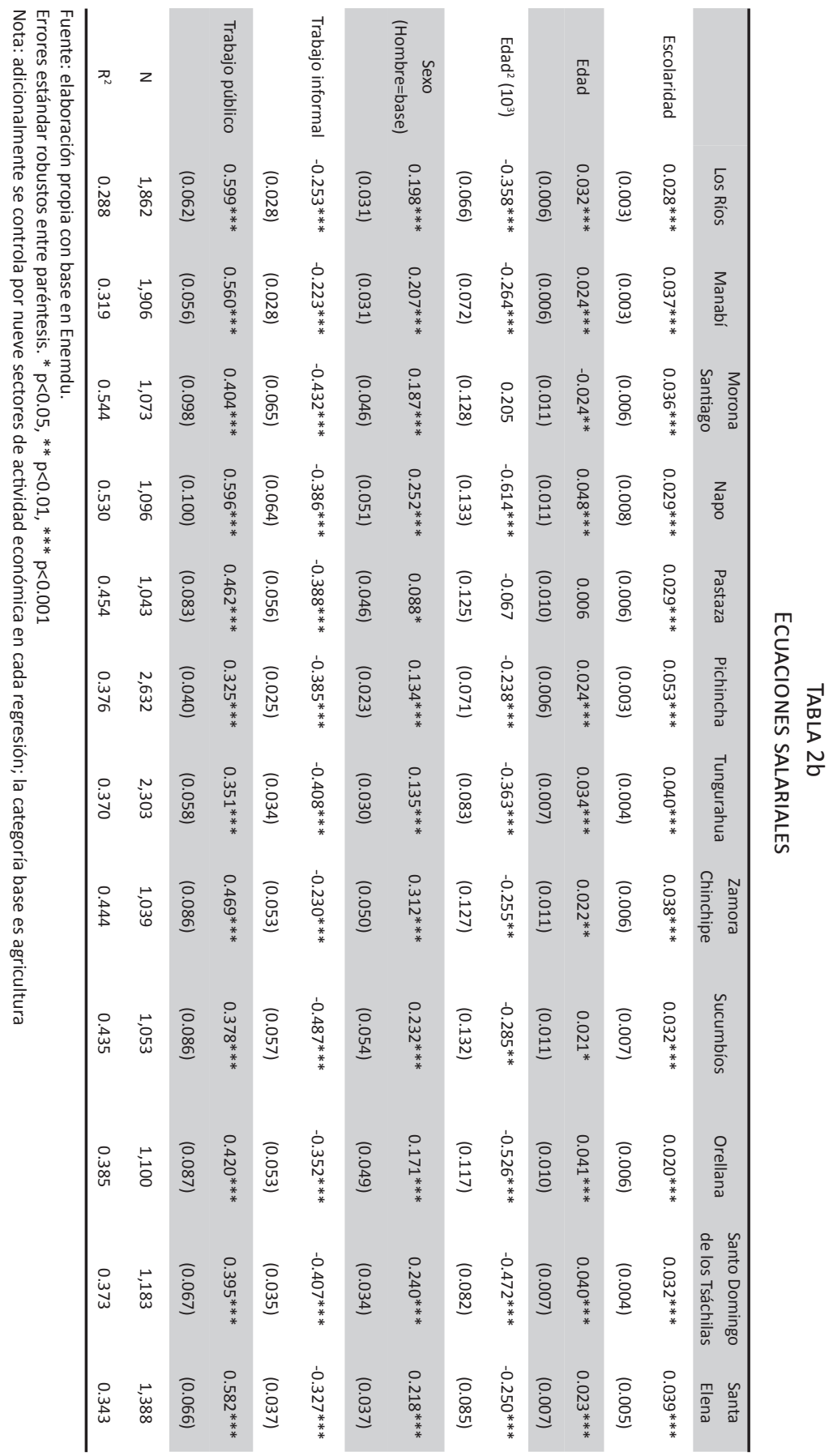


Con referencia a las regresiones cuantílicas, los resultados se presentan para los cuartiles de la distribución. Debido al elevado número de coeficientes para los cuartiles de las 23 provincias analizadas, el estudio sólo presenta los efectos de las variables analizadas. Los resultados se muestran en la Tabla 3. En general, los coeficientes estimados a lo largo de la distribución del ingreso laboral son estadísticamente significativos y presentan los efectos esperados para todas las provincias.

Se observa un patrón heterogéneo de los retornos a la educación a lo largo de la distribución del ingreso laboral. El retorno nacional estimado es de $2.98 \%$ en el primer cuartil, $2.56 \%$ en el medio de la distribución, y $5.72 \%$ en el tercer cuartil. En este sentido, el incremento del ingreso laboral causado por un año adicional de educación es el doble en la parte superior de la distribución que en la inferior. Se observa un patrón similar en todas las provincias. Adicionalmente, se determina que en algunas provincias el retorno a la educación en la parte media de la distribución del ingreso laboral es menor que en la baja. Por lo tanto, el incremento del nivel de educación de la población en estas regiones provoca una disminución de la desigualdad del ingreso laboral en la parte media-baja de la distribución, debido a que beneficia principalmente a los trabajadores de menores ingresos.

Los resultados muestran que, en general, el efecto de la informalidad laboral disminuye a lo largo de la distribución salarial. Los resultados evidencian que la penalidad salarial es de aproximadamente $58.6 \%$ para los trabajadores con los más bajos ingresos, $36.4 \%$ para los de ingresos promedio, y $27.2 \%$ para los de mayores ingresos. Cabe señalar que la penalidad salarial de la informalidad laboral en los trabajadores de altos ingresos es negativa y estadísticamente significativa en la mayoría de las provincias. En este sentido, los resultados sugieren que el incremento de la informalidad laboral contribuirá a incrementar la desigualdad dentro de las provincias, reduciendo los ingresos laborales de los trabajadores de menores ingresos más que para los trabajadores de altos ingresos. No obstante, el tamaño de este efecto varía considerablemente entre las regiones

Las estimaciones señalan que en el caso del trabajo del sector público, la prima salarial incrementa a lo largo de la distribución salarial. El efecto estimado en el país en su conjunto es de $11.2 \%$ en el primer cuartil, incrementa $24.5 \%$ en el medio de la distribución, y aumenta marcadamente $105.6 \%$ en el tercer cuartil. Un patrón similar 
se observa en la mayor parte del país, excepto en algunas provincias, en las cuales el efecto del trabajo en el sector público no es significativo para los trabajadores de menos ingresos. Cabe señalar que la prima salarial del trabajo en el sector público a lo largo de la distribución varía considerablemente entre regiones.

TABLA 3

COEFICIENTES ESTIMADOS POR PERCENTIL PARA EDUCACIÓN, INFORMALIDAD, Y EMPLEO PÚBLICO. REGRESIÓN CUANTIL INCONDICIONAL

\begin{tabular}{|c|c|c|c|c|c|c|c|c|c|}
\hline \multirow[t]{3}{*}{ Región } & \multicolumn{3}{|c|}{ Educación } & \multicolumn{3}{|c|}{ Informalidad laboral } & \multicolumn{3}{|c|}{ Trabajo público } \\
\hline & \multicolumn{9}{|c|}{ Percentil } \\
\hline & 25 & 50 & 75 & 25 & 50 & 75 & 25 & 50 & 75 \\
\hline \multirow[t]{2}{*}{ Nacional } & $0.0298^{* * *}$ & $0.0256^{* * *}$ & $0.0572^{* * *}$ & $-0.586 * * *$ & $-0.364^{* * *}$ & $-0.272^{* * *}$ & $0.112^{* * *}$ & $0.245^{* * *}$ & $1.056^{* * *}$ \\
\hline & $(0.00212)$ & $(0.00117)$ & (0.00199) & $(0.0177)$ & $(0.0107)$ & $(0.0169)$ & $(0.0178)$ & $(0.0130)$ & $(0.0312)$ \\
\hline \multirow[t]{2}{*}{ Azuay } & $0.0512^{* * *}$ & $0.0342^{* * *}$ & $0.0715^{* * *}$ & $-0.813^{* * *}$ & $-0.459^{* * *}$ & $-0.347^{* * *}$ & -0.00615 & $0.268^{* * *}$ & $0.927^{* * *}$ \\
\hline & $(0.0106)$ & $(0.00487)$ & $(0.00769)$ & $(0.0983)$ & $(0.0483)$ & $(0.0643)$ & $(0.0765)$ & $(0.0540)$ & $(0.134)$ \\
\hline \multirow[t]{2}{*}{ Bolívar } & -0.00357 & 0.00732 & $0.0352^{* * *}$ & $-0.274 * *$ & $-0.223^{* *}$ & $-0.307 * *$ & 0.0688 & 0.130 & $1.051^{* * *}$ \\
\hline & $(0.00981)$ & $(0.00824)$ & (0.00968) & (0.0839) & $(0.0787)$ & $(0.106)$ & $(0.0779)$ & $(0.0808)$ & $(0.224)$ \\
\hline \multirow[t]{2}{*}{ Cañar } & $0.0302^{*}$ & $0.0218^{* * *}$ & $0.0565^{* * *}$ & $-0.374 * *$ & $-0.296 * * *$ & $-0.207^{*}$ & $0.264^{*}$ & $0.221 * * *$ & $0.711^{* * *}$ \\
\hline & (0.0126) & $(0.00629)$ & $(0.0103)$ & $(0.122)$ & $(0.0703)$ & $(0.0851)$ & $(0.102)$ & $(0.0559)$ & $(0.162)$ \\
\hline \multirow[t]{2}{*}{ Carchi } & $0.0340^{* * *}$ & $0.0232^{*}$ & $0.0384^{* * *}$ & $-0.333^{* * *}$ & $-0.452^{* * *}$ & $-0.154^{*}$ & 0.208 & $0.530^{* * *}$ & $1.256^{* * *}$ \\
\hline & (0.0103) & $(0.00939)$ & $(0.00901)$ & $(0.0762)$ & (0.0858) & $(0.0716)$ & $(0.118)$ & $(0.129)$ & (0.133) \\
\hline \multirow[t]{2}{*}{ Cotopaxi } & $0.0335^{* * *}$ & $0.0251^{* * *}$ & $0.0346 * * *$ & $-0.555^{* * *}$ & $-0.294 * * *$ & $-0.165^{*}$ & 0.103 & $0.308^{* * *}$ & $1.378^{* * *}$ \\
\hline & $(0.00786)$ & $(0.00479)$ & $(0.00752)$ & $(0.0663)$ & $(0.0451)$ & $(0.0667)$ & $(0.0940)$ & $(0.0759)$ & $(0.128)$ \\
\hline \multirow[t]{2}{*}{ Chimborazo } & 0.0157 & 0.0117 & $0.0358^{* *}$ & $-0.283^{*}$ & $-0.409 * * *$ & $-0.269 * *$ & $0.494^{*}$ & $0.511^{* * *}$ & $1.664^{* * *}$ \\
\hline & $(0.0154)$ & $(0.00983)$ & $(0.0124)$ & $(0.143)$ & $(0.0841)$ & $(0.100)$ & $(0.246)$ & (0.145) & $(0.202)$ \\
\hline \multirow[t]{2}{*}{ El Oro } & $0.0413^{* * *}$ & $0.0208^{* * *}$ & $0.0360^{* * *}$ & $-0.646^{* * *}$ & $-0.369^{* * *}$ & $-0.208^{* * *}$ & 0.0254 & $0.270^{* * *}$ & $0.709^{* * *}$ \\
\hline & $(0.00846)$ & $(0.00401)$ & $(0.00524)$ & $(0.0577)$ & (0.0368) & $(0.0486)$ & $(0.0648)$ & $(0.0465)$ & $(0.0951)$ \\
\hline \multirow[t]{2}{*}{ Esmeraldas } & $0.0358^{* *}$ & $0.0239 * *$ & $0.0549 * * *$ & $-0.631^{* * *}$ & $-0.538^{* * *}$ & $-0.269 * * *$ & 0.145 & $0.475^{* * *}$ & $1.315^{* * *}$ \\
\hline & $(0.0120)$ & $(0.00778)$ & $(0.00866)$ & $(0.115)$ & $(0.0771)$ & $(0.0693)$ & $(0.108)$ & $(0.0902)$ & $(0.136)$ \\
\hline \multirow[t]{2}{*}{ Guayas } & $0.0306^{* * *}$ & $0.0298^{* * *}$ & $0.0472^{* * *}$ & $-0.542^{* * *}$ & $-0.388^{* * *}$ & $-0.331^{* * *}$ & 0.0498 & $0.283^{* * *}$ & $1.019^{* * *}$ \\
\hline & $(0.00425)$ & $(0.00298)$ & (0.00443) & $(0.0334)$ & (0.0255) & $(0.0360)$ & $(0.0336)$ & $(0.0311)$ & $(0.0648)$ \\
\hline \multirow[t]{2}{*}{ Imbabura } & $0.0301 * * *$ & $0.0235^{* * *}$ & $0.0578^{* * *}$ & $-0.616^{* * *}$ & $-0.425 * * *$ & $-0.189 * * *$ & $0.220^{* *}$ & $0.274^{* * *}$ & $1.333^{* * *}$ \\
\hline & $(0.00643)$ & $(0.00385)$ & $(0.00634)$ & (0.0583) & $(0.0390)$ & $(0.0562)$ & $(0.0706)$ & $(0.0474)$ & $(0.108)$ \\
\hline
\end{tabular}


Percentil

\begin{tabular}{|c|c|c|c|c|c|c|c|c|c|}
\hline & 25 & 50 & 75 & 25 & 50 & 75 & 25 & 50 & 75 \\
\hline \multirow[t]{2}{*}{ Loja } & $0.0610^{* * *}$ & $0.0298^{* * *}$ & $0.0567 * * *$ & $-0.571 * * *$ & $-0.528 * * *$ & -0.138 & 0.228 & $0.437 * * *$ & $0.977^{* * *}$ \\
\hline & $(0.0150)$ & $(0.00839)$ & $(0.00904)$ & $(0.133)$ & $(0.0846)$ & $(0.0763)$ & $(0.156)$ & $(0.104)$ & $(0.159)$ \\
\hline \multirow[t]{2}{*}{ Los Ríos } & $0.0162 * *$ & $0.0228^{* * *}$ & $0.0335^{* * *}$ & $-0.330 * * *$ & $-0.308^{* * *}$ & $-0.204 * * *$ & $0.210^{* *}$ & $0.313^{* * *}$ & $0.956 * * *$ \\
\hline & $(0.00565)$ & $(0.00412)$ & $(0.00542)$ & $(0.0465)$ & $(0.0380)$ & $(0.0465)$ & $(0.0696)$ & $(0.0510)$ & $(0.0806)$ \\
\hline \multirow[t]{2}{*}{ Manabí } & $0.0274^{* * *}$ & $0.0353^{* * *}$ & $0.0381^{* * *}$ & $-0.263^{* * *}$ & $-0.380^{* * *}$ & $-0.117^{* *}$ & $0.215^{* * *}$ & $0.365^{* * *}$ & $0.965 * * *$ \\
\hline & $(0.00580)$ & $(0.00513)$ & $(0.00526)$ & $(0.0496)$ & $(0.0462)$ & $(0.0452)$ & $(0.0581)$ & $(0.0566)$ & $(0.0883)$ \\
\hline \multirow{2}{*}{$\begin{array}{l}\text { Morona } \\
\text { Santiago }\end{array}$} & 0.0244 & $0.0246^{*}$ & $0.0735^{* * *}$ & $-0.706 * * *$ & $-0.909 * * *$ & -0.113 & 0.304 & $0.509^{*}$ & $0.792 * * *$ \\
\hline & (0.0195) & $(0.0117)$ & (0.0106) & $(0.200)$ & $(0.146)$ & $(0.0884)$ & $(0.246)$ & $(0.211)$ & $(0.182)$ \\
\hline \multirow[t]{2}{*}{ Napo } & 0.0128 & 0.0204 & $0.0432 * * *$ & -0.378 & $-0.803^{* * *}$ & $-0.352^{* * *}$ & 0.394 & $0.658^{* * *}$ & $1.306^{* * *}$ \\
\hline & $(0.0206)$ & $(0.0141)$ & $(0.0115)$ & $(0.220)$ & $(0.143)$ & $(0.0996)$ & $(0.203)$ & $(0.166)$ & (0.193) \\
\hline \multirow[t]{2}{*}{ Pastaza } & 0.0143 & $0.0261^{* *}$ & $0.0494^{* * *}$ & $-0.733^{* * *}$ & $-0.551^{* * *}$ & $-0.284^{* *}$ & 0.193 & $0.492^{* * *}$ & $1.045^{* * *}$ \\
\hline & $(0.0156)$ & $(0.00916)$ & $(0.00982)$ & $(0.122)$ & $(0.104)$ & $(0.0912)$ & (0.139) & (0.141) & (0.136) \\
\hline \multirow[t]{2}{*}{ Pichincha } & $0.0223^{* * *}$ & $0.0355^{* * *}$ & $0.0809^{* * *}$ & $-0.571^{* * *}$ & $-0.289^{* * *}$ & $-0.166^{* * *}$ & $0.0778^{*}$ & $0.288^{* * *}$ & $0.838^{* * * *}$ \\
\hline & $(0.00435)$ & $(0.00372)$ & $(0.00593)$ & $(0.0400)$ & (0.0316) & $(0.0494)$ & $(0.0305)$ & $(0.0358)$ & $(0.0905)$ \\
\hline \multirow[t]{2}{*}{ Tungurahua } & $0.0222^{*}$ & $0.0341^{* * *}$ & $0.0698^{* * *}$ & $-0.611^{* * *}$ & $-0.519 * * *$ & $-0.215^{* *}$ & 0.00107 & $0.173^{*}$ & $0.942^{* * *}$ \\
\hline & $(0.00949)$ & $(0.00697)$ & $(0.0100)$ & (0.0684) & $(0.0564)$ & $(0.0781)$ & $(0.0606)$ & $(0.0780)$ & (0.156) \\
\hline \multirow{2}{*}{$\begin{array}{c}\text { Zamora } \\
\text { Chinchipe }\end{array}$} & 0.0266 & $0.0284^{* *}$ & $0.0616^{* * *}$ & -0.205 & $-0.460^{* * *}$ & $-0.245^{* *}$ & 0.158 & $0.513^{* * *}$ & $0.928^{* * *}$ \\
\hline & $(0.0157)$ & $(0.00889)$ & $(0.0116)$ & (0.139) & $(0.0813)$ & $(0.0801)$ & $(0.185)$ & $(0.121)$ & (0.174) \\
\hline \multirow[t]{2}{*}{ Sucumbíos } & 0.00750 & $0.0264^{* *}$ & $0.0620^{* * *}$ & $-0.459 * * *$ & $-0.894^{* * *}$ & $-0.274^{* *}$ & $0.265^{* *}$ & $0.370^{* * *}$ & $0.870^{* * *}$ \\
\hline & (0.0138) & $(0.00961)$ & (0.00994) & (0.118) & (0.0988) & $(0.0879)$ & $(0.0939)$ & (0.106) & (0.170) \\
\hline \multirow[t]{2}{*}{ Orellana } & -0.0123 & $0.0241^{* *}$ & $0.0468^{* * *}$ & $-0.335^{* *}$ & $-0.514^{* * *}$ & $-0.434 * * *$ & $0.422^{* *}$ & $0.505^{* * *}$ & $0.524^{* *}$ \\
\hline & $(0.0122)$ & (0.00809) & $(0.00983)$ & (0.107) & $(0.0788)$ & (0.0896) & (0.130) & (0.109) & (0.170) \\
\hline \multirow{3}{*}{$\begin{array}{c}\text { Santo } \\
\text { Domingo de } \\
\text { los Tsáchilas }\end{array}$} & & & & & & & & & \\
\hline & 0.0144 & $0.0195^{* * *}$ & $0.0501^{* * *}$ & $-0.504^{* * *}$ & $-0.500^{* * *}$ & $-0.408^{* * *}$ & 0.00597 & $0.132^{*}$ & $1.001^{* * *}$ \\
\hline & $(0.00751)$ & $(0.00525)$ & $(0.00671)$ & $(0.0631)$ & $(0.0462)$ & $(0.0675)$ & $(0.0633)$ & $(0.0523)$ & (0.101) \\
\hline \multirow[t]{2}{*}{ Santa Elena } & $0.0371^{* * *}$ & $0.0293^{* * *}$ & $0.0426^{* * *}$ & $-0.525 * * *$ & $-0.459 * * *$ & $-0.170^{* *}$ & 0.0363 & $0.214^{* * *}$ & $0.966 * * *$ \\
\hline & $(0.00788)$ & $(0.00530)$ & (0.00759) & (0.0656) & (0.0508) & $(0.0585)$ & $(0.0702)$ & $(0.0615)$ & (0.108) \\
\hline
\end{tabular}

Fuente: elaboración propia con base en la Enemdu.

Errores estándar robustos entre paréntesis. ${ }^{*} p<0.05, * * p<0.01, * * * p<0.001$

Nota: se incluye como controles edad (y su cuadrado), género y sector de actividad económica.

A modo de conclusión, los resultados muestran marcadas diferencias regionales en los retornos a la educación. Asimismo, las estimaciones revelan que los trabajadores informales enfrentan diferentes penalidades salariales entre las provincias y a lo largo de la distribución del ingreso laboral, y que esta penalidad salarial afecta principalmente a los 
trabajadores de menores ingresos. Igualmente, los resultados confirman el efecto positivo del trabajo público sobre los ingresos laborales, el cual incrementa a lo largo de la distribución del ingreso laboral, y la existencia de importantes diferencias regionales en las primas salariales asociadas a este tipo la brecha de trabajo.

\subsection{Descomposición de las brechas salariales regionales}

La descomposición salarial entre provincias se analiza considerando la diferencia entre Pichincha, la de más alto nivel de ingreso laboral promedio, y las otras regiones. La descomposición respecto a esta provincia para la media se presenta en la Tablas $4 \mathrm{a}$ y $4 \mathrm{~b}$ y para los cuartiles analizados en las Figuras A.4 y A.5 del anexo.

En todos los casos, las brechas salariales entre Pichincha y el resto de regiones son estadísticamente significativas. Éstas se pueden explicar como una combinación de diferencias entre características y estructura salarial. La mayor brecha de ingreso laboral se encuentra en la provincia de Napo, mientras que la menor es la de Guayas. No obstante, las regiones difieren en el origen de las brechas.

Por ejemplo, en Napo, 0.116 puntos logarítmicos (p.1.) de los 0.67 p.1. de la brecha salarial se define por diferencias en las características entre esta provincia y Pichincha. De forma semejante, en Santo Domingo de los Tsáchilas, la parte de la brecha salarial justificada por diferencias en características llega a representar 0.188 p.1. de 0.299 p.l. En contraste, solamente 0.059 p.l. de 0.401 p.l. de la brecha salarial entre Loja y Pichincha se aclara por diferencias en características. Los resultados señalan que los bajos ingresos laborales en las provincias de Cañar, Cotopaxi, El Oro, Los Ríos, Tungurahua, Santo Domingo de los Tsáchilas y Santa Elena se explican principalmente por su menor dotación de factores que contribuyen a incrementar los ingresos laborales. En el caso de las provincias de Azuay, Bolívar, Carchi, Chimborazo, Esmeraldas, Guayas, Imbabura, Loja, Manabí, Morona Santiago, Napo, Pastaza, Zamora Chinchipe, Sucumbíos y Orellana, la brecha de ingreso se justifica principalmente por diferencias en la estructura salarial.

La descomposición detallada señala que las diferencias regionales en los ingresos laborales se explican principalmente por disparidades en los niveles de educación del empleo. Los resultados también muestran que las discrepancias en la incidencia de la informalidad laboral 
contribuyen a determinar la diversidad en los ingresos laborales entre regiones. Asimismo, el empleo en el sector público explica una parte de la desigualdad regional, aunque en menor medida. Cabe señalar que, en la mayoría de los casos, la informalidad contribuye a incrementar la brecha de ingreso entre regiones, mientras que el empleo público contribuye a reducir la desigualdad. No obstante, la importancia de cada factor varía considerablemente entre provincias. Por ejemplo, 0.322 p.l. de la brecha salarial promedio entre Napo y Pichincha se explica por el mayor nivel de educación del empleo en esta última provincia, 0.075 p.1., la incidencia de la informalidad laboral, y -0.095, empleo público. El signo negativo en este último coeficiente indica que en ausencia de éste, la brecha salarial entre estas regiones sería aún mayor. Adicionalmente, en las provincias en las cuales la brecha salarial es pequeña, la contribución de la educación disminuye, pero incrementa la importancia de la informalidad laboral y el empleo público.

Para simplificar el análisis se tomarán como referencia dos provincias, una con ingreso promedio alto, Guayas, y una con ingreso promedio bajo, Napo. Las diferencias en las brechas salariales a lo largo de la distribución del ingreso laboral se presentan en la Tabla A.2 del anexo. En general, las brechas siguen la forma de una U, o disminuyen a lo largo de la distribución. Si tomamos como referencia la provincia de Guayas, se observa que la brecha salarial es de 0.217 p.l., 0.143 p.l. y 0.273 p.l. en el primero, segundo y tercer cuartil, respectivamente. En el caso de la provincia de Napo, la brecha disminuye de 0.795 p.l., en el primer cuartil, a 0.133 p.l., en el tercer cuartil.

La descomposición de la brecha salarial a lo largo de la distribución salarial señala que las diferencias en características entre regiones tienen un efecto principalmente en el primer y tercer cuartil, mientras que las diferencias en la estructura salarial afectan la brecha salarial, principalmente en la parte superior de la distribución.

La descomposición detallada de los efectos composición y precio para los diferentes puntos de la distribución del ingreso laboral se presenta en las Figuras A.4 y A.5 del anexo. Los resultados muestran que las diferencias entre regiones en el nivel de educación, informalidad laboral y empleo público generan brechas de ingreso a lo largo de la distribución del ingreso laboral. Por un lado, las diferencias regionales en el nivel de educación generan disparidades salariales principalmente en la parte superior de la distribución. Por otro, las discrepancias regio- 
nales asociadas a la informalidad laboral provocan disparidades salariales en la parte media-baja de la distribución, mientras que el empleo público explica una parte importante de la brecha salarial en la parte media-alta de la distribución.

En el caso de la educación, si se toma como referencia la provincia de Guayas, las diferencias regionales en la dotación de educación explican 0.0451 p.l. en el tercer cuartil, pero sólo 0.0120 p.l. en el primero. En el caso de Napo se observa algo semejante, 0.052 p.l. en el tercer cuartil y 0.014 p.l. en el primero.

Los resultados también señalan que las diferencias en los retornos a la educación determinan las brechas salariales regionales en la parte media-alta de la distribución: 0.542 p.l. en el tercer cuartil y -0.114 p.l. en el primero en Guayas, y 0.458 p.l. en el tercer cuartil y 0.233 en el primero en Napo. De esta forma, el efecto conjunto atribuible a las diferencias en la dotación y en el retorno a la educación señala que las diferencias regionales asociadas al capital humano explican una parte importante de las brechas de ingreso regionales, principalmente en el extremo superior de la distribución.

Respecto al efecto de la informalidad laboral, se determina un patrón inverso al presentado por la educación. En general, se presenta principalmente en la parte media de la distribución. Si se toma como referencia la provincia de Guayas, las diferencias en la incidencia de la informalidad laboral explican 0.118 p.l. en el primer cuartil, 0.046 en el medio, y 0.0314 en la parte superior de la distribución salarial. No obstante, el efecto de las diferencias regionales en la penalidad salarial de la informalidad presenta un comportamiento más complejo. En este caso, las diferencias regionales en la penalidad salarial contribuyen a disminuir la brecha salarial regional en el primer cuartil (-0.098 p.1.), pero la incrementa en el segundo y tercero (0.038 p.l. y 0.051$)$. El efecto total asociado a la informalidad laboral es de 0.021 p.l., 0.084 p.l. y 0.0820 p.l. en el primero, segundo y tercer cuartil, respectivamente. En la provincia de Napo se observa un comportamiento similar.

En la mayoría de las regiones, el impacto del empleo público se observa principalmente en la parte media-alta de la distribución. En el caso de Guayas, el efecto de las diferencias regionales en la tasa de empleo público es de 0.025 p.l. en el tercer cuartil y de 0.003 p.l. en el primero. De igual forma, las diferencias regionales en la prima salarial del sector público explican 0.004 p.l. de la brecha salarial regional en el 
primer cuartil y -0.028 p.l. en el tercero. En Napo se observa un patrón similar; la diferencia en la tasa de empleo público explica -0.016 p.l. y -0.117 p.l. en el primero y tercer cuartil, respectivamente, y las diferencias en la prima salarial del empleo público en -0.066 p.l y -0.085 p.l. en el primero y tercer cuartil, respectivamente.

Resumiendo, la descomposición muestra que las diferencias regionales en el nivel de educación, informalidad laboral, así como empleo público explican una parte importante de la brecha en los ingresos laborales entre regiones. Los resultados también señalan que las diferencias regionales en el nivel de educación afectan principalmente la parte superior de la distribución del ingreso laboral; las asociadas a la informalidad laboral explican las brechas en la parte media de la distribución. Por su parte, el empleo público explica una parte importante de la brecha de ingreso en la parte media-alta de la distribución. Finalmente se debe señalar que los resultados varían notablemente entre provincias. 


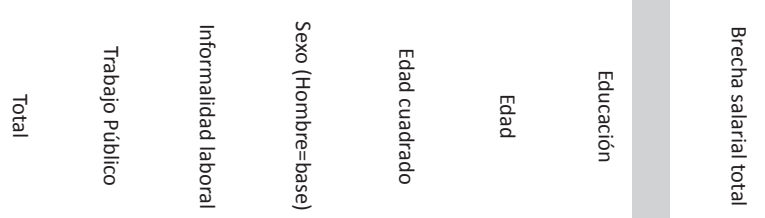

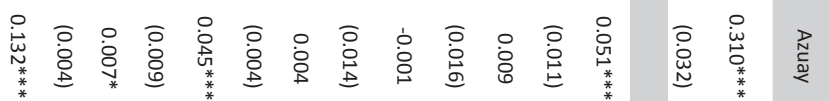

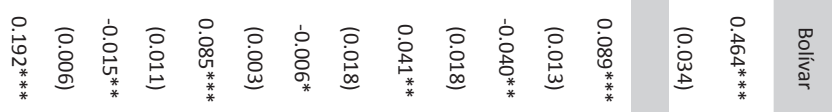

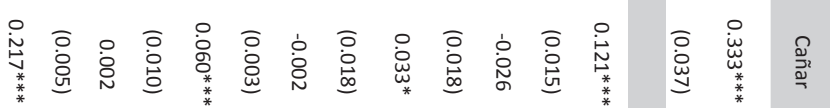

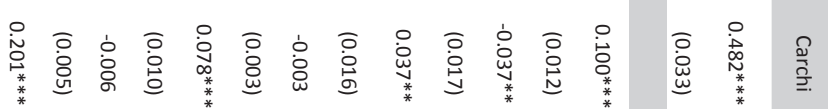

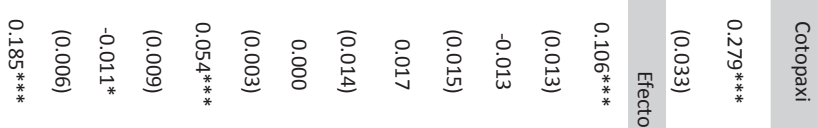

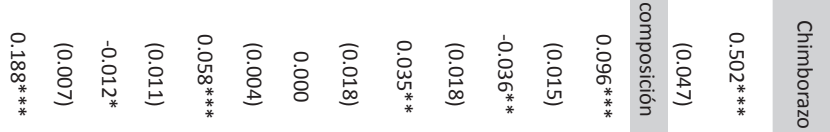

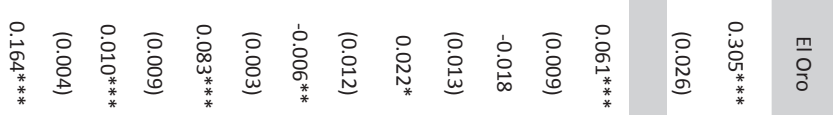

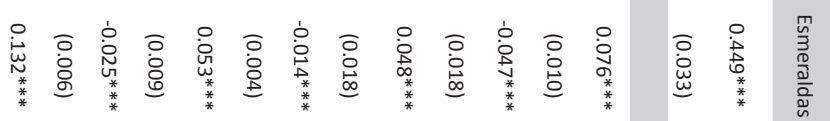

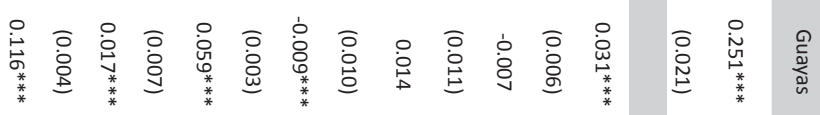

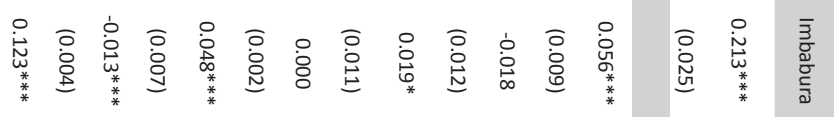

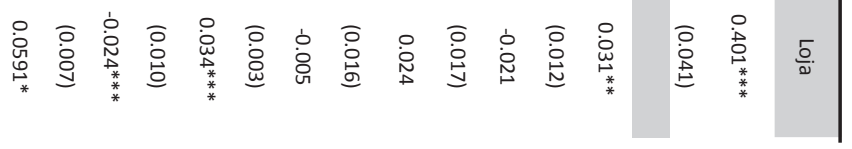




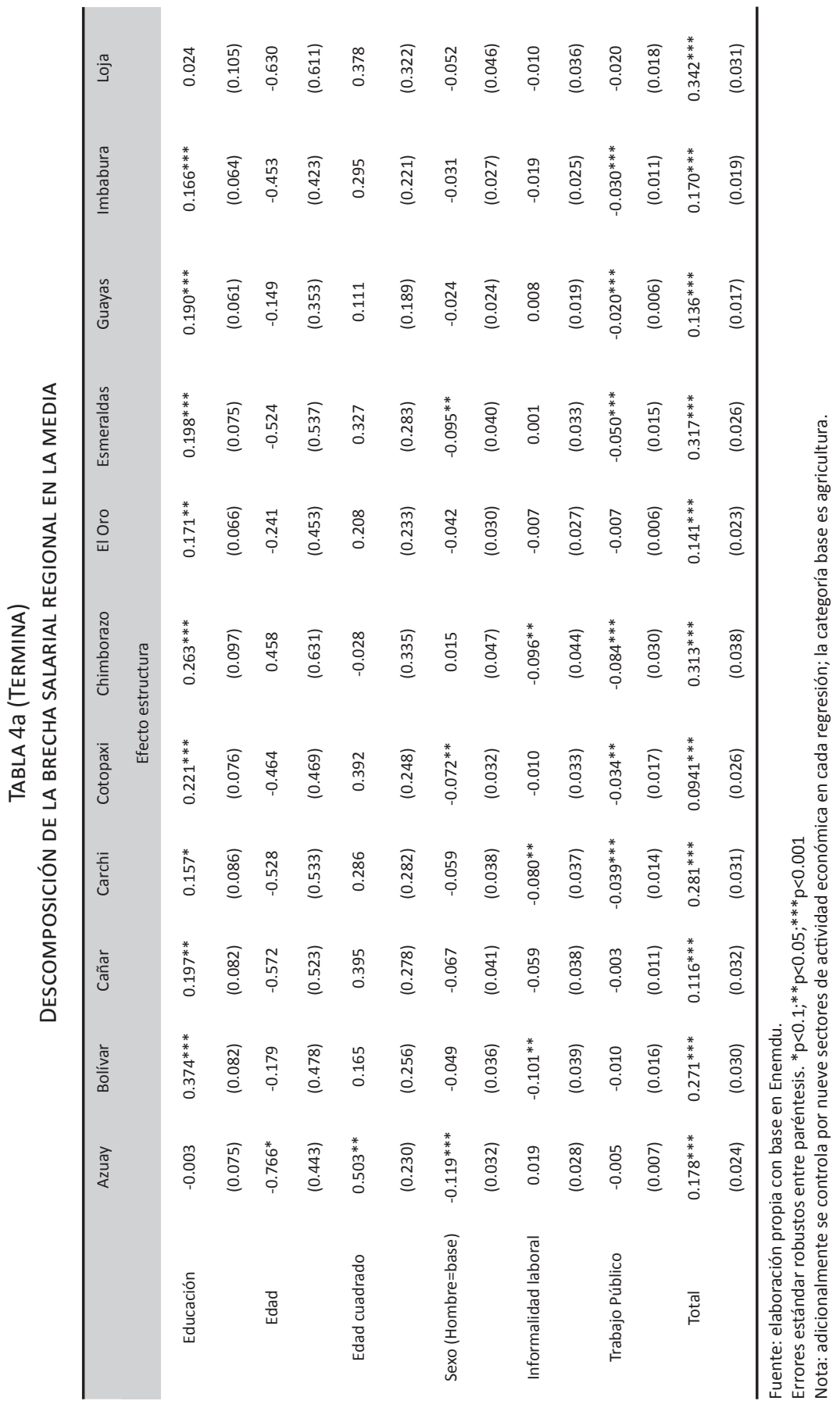




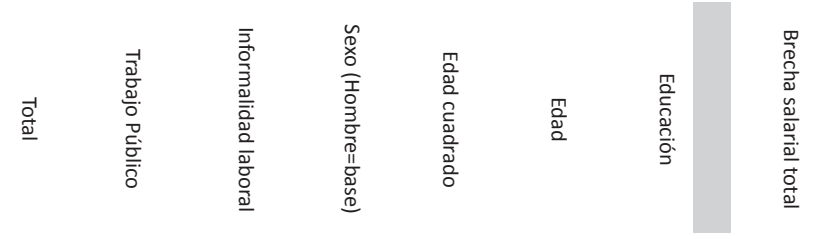

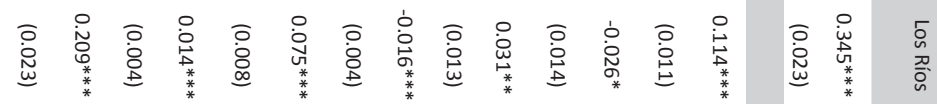

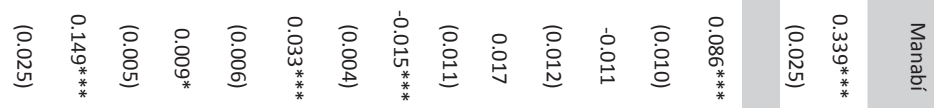

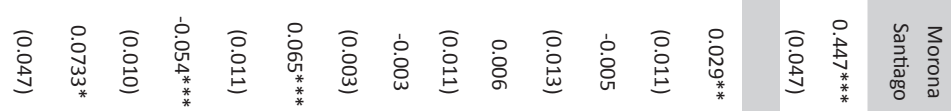

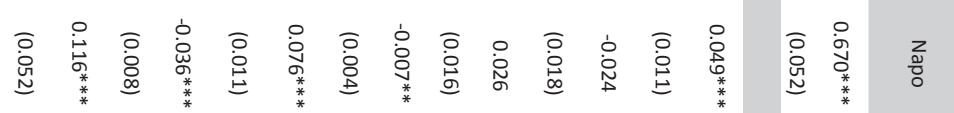

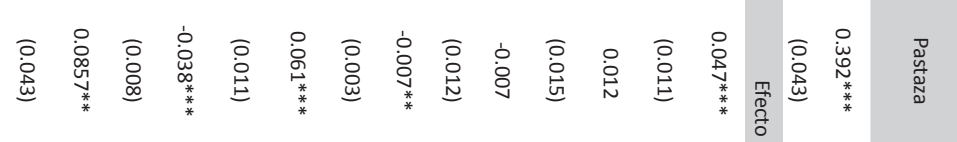

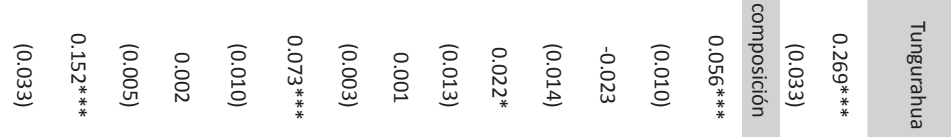

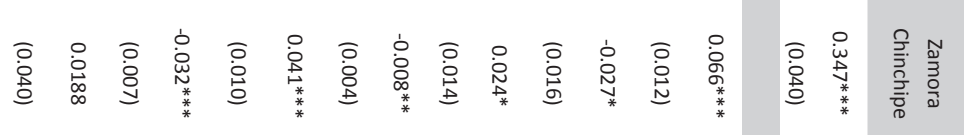

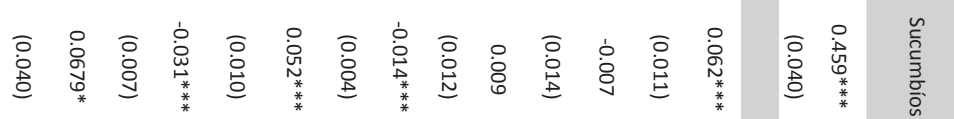

怘

은

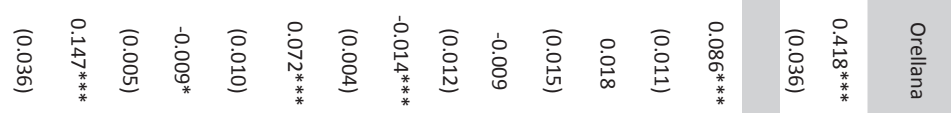

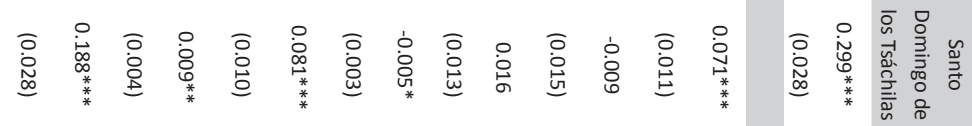

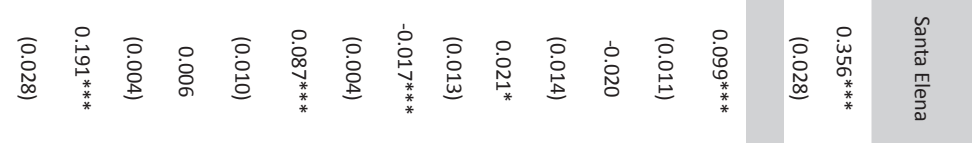




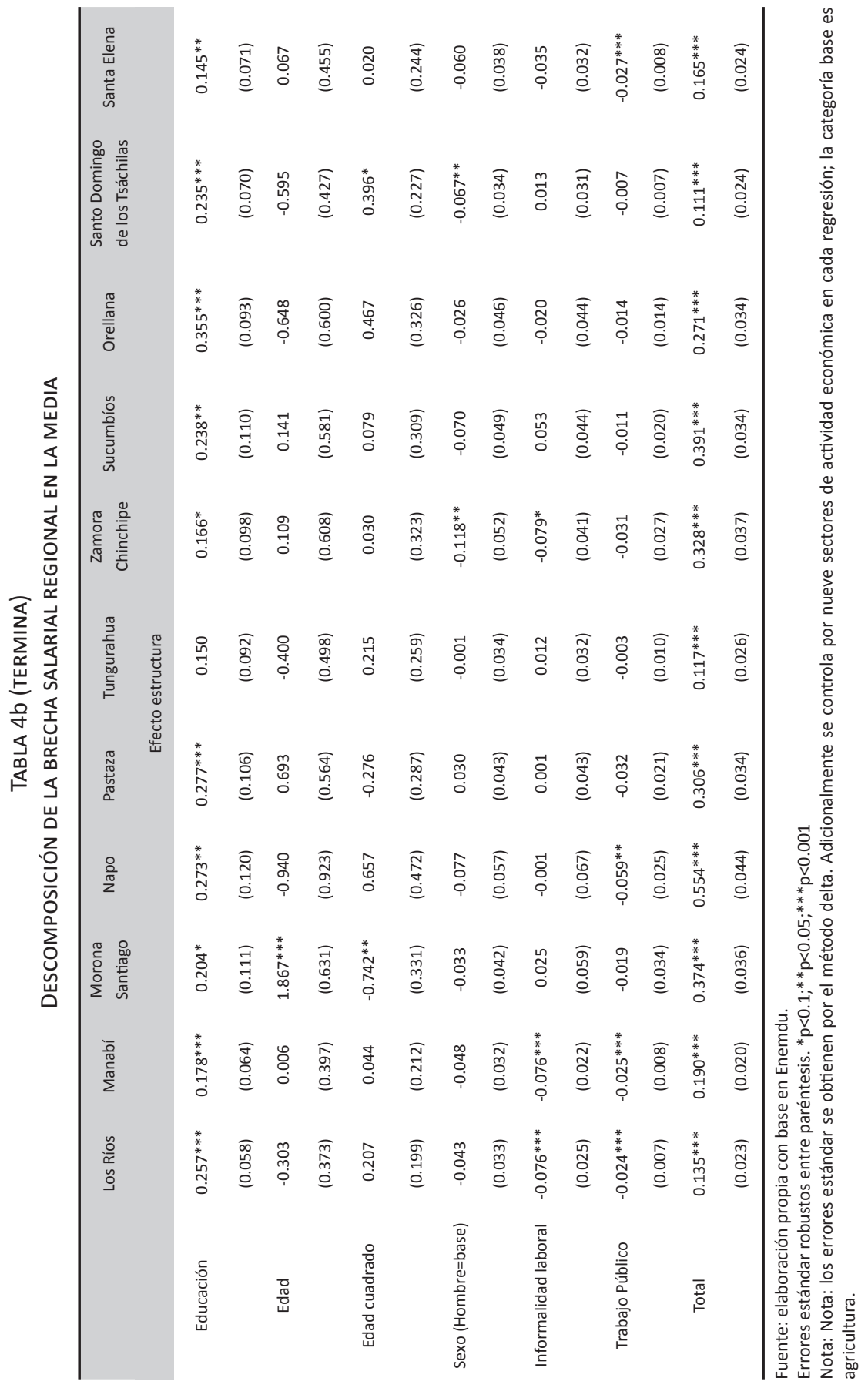




\section{CONCLUSIONES}

En el presente estudio se analizaron los determinantes de las brechas regionales de ingreso laboral en Ecuador, específicamente la importancia de la educación, la informalidad laboral y el empleo público. Con base en métodos de descomposición basados en regresiones de función de influencia recentrada, se determinó el impacto cuantitativo de cada una de las variables explicativas sobre la brecha regional de ingresos laborales. Parte de la diferencia regional de ingresos laborales se atribuye a discrepancias en las características de los trabajadores, así como a diferencias en la estructura salarial entre regiones. Se aplicó la descomposición estándar de Oaxaca-Blinder para aislar la contribución de la educación, informalidad laboral y empleo público sobre la brecha de ingreso en la media y diferentes cuantiles.

Los resultados señalan que existen marcadas diferencias entre regiones en términos de ingresos laborales. La provincia de menor ingreso tiene un nivel promedio que representa el 53\% la de mayor ingreso. Asimismo, hay marcadas discrepancias regionales en la distribución regional del capital humano, informalidad laboral y empleo público. También, se observa una heterogeneidad en la estructura sectorial de las regiones.

Las estimaciones obtenidas mediante la regresión cuantílica incondicional muestran un patrón heterogéneo. En el caso de la educación, los retornos estimados incrementan a lo largo de la distribución del ingreso laboral. El efecto de la informalidad laboral disminuye para los cuantiles más altos. La prima salarial del sector público incrementa a lo largo de la distribución del ingreso laboral.

En todos los casos las brechas salariales entre las provincias son estadísticamente significativas; las disparidades de ingreso regionales se pueden explicar como una combinación de diferencias en características y estructura salarial. Respecto a las diferencias regionales en los ingresos laborales, es posible explicarlas principalmente por discrepancias en los niveles de educación del empleo. La informalidad laboral y empleo en el sector público explican una parte importante de la desigualdad regional de ingresos, aunque en menor medida.

La descomposición de la brecha de ingreso regional a lo largo de la distribución señala que las diferencias regionales en el nivel de educación generan disparidades principalmente en la parte superior de 
la distribución; la informalidad laboral genera diferencias de ingreso particularmente en la parte media-baja de la distribución, y el empleo público explica una parte importante de la brecha de ingreso en la parte media-alta de la distribución. No obstante, se observa una marcada heterogeneidad regional en los resultados.

El presente estudio muestra que la desigualdad del ingreso laboral en Ecuador es un fenómeno que requiere el análisis de diferentes tipos de dimensiones y determinantes. Los resultados sugieren que las diferencias regionales en la composición y estructura salarial del trabajo generan discrepancias en los niveles de ingreso entre las regiones, mismas que responden en gran medida a diferencias regionales en educación, informalidad laboral y empleo público.

Asimismo, se sugieren implicaciones de política que pueden ayudar a reducir las brechas regionales de ingreso. Se deben continuar implementando políticas educativas para garantizar el acceso a la educación y generar incentivos necesarios para que las generaciones jóvenes continúen sus estudios, particularmente en las regiones económicamente menos dinámicas. Es imperioso generar empleo formal y mayor control de la normativa laboral que contribuya a reducir la informalidad laboral que afecta negativamente los ingresos de los trabajadores y repercute en la brecha de ingreso regional. Se debe considerar que las diferencias regionales en la composición y tasas salariales del empleo público explican una parte importante de las discrepancias regionales de ingreso.

Los resultados en este estudio tienen, por supuesto, algunas limitaciones. Primero, la endogeneidad de los retornos a la educación causada por características no observadas, como las habilidades de los trabajadores que afectan los ingresos laborales. Si los trabajadores difieren en sus habilidades, y éstas afectan sus ingresos, los retornos a la educación estimados estarán sesgados. Este problema se puede abordar mediante el método de variables instrumentales; no obstante, esto no es posible debido a la falta de instrumentos apropiados. Segundo, el sesgo de selección debido a que la decisión de participar en el mercado laboral puede no estar asignada aleatoriamente. En los resultados presentados no se controla por sesgo de selección debido a que cuando se realizaron las estimaciones mediante ello, los resultados fueron similares, ${ }^{7}$ aunque en

\footnotetext{
Los resultados de las ecuaciones salariales controlando por sesgo de selección mediante un
} modelo de selección de Heckman se presentan en las Tablas A.3a y A.3b del anexo 
otros estudios aplicados para Ecuador se señala la necesidad de corregir el sesgo de selección. A pesar de que estos problemas de estimación pueden afectar los resultados, las estimaciones presentadas demuestran una primera aproximación al estudio de las brechas de ingreso laboral entre las provincias ecuatorianas. Adicionalmente, un aspecto que no se abordó en este estudio es la importancia de las externalidades de aglomeración en la determinación de las diferencias salariales regionales. Ciertamente, estos factores deben ser considerados en estudios posteriores.

\section{BIBLIOGRAFÍA}

Alejo, J., y C. Parada (2017), "Desigualdad e informalidad en América Latina: el caso de Brasil", Desarrollo y Sociedad, (78), 143-199.

Amarante, V. (2001), "Diferencias salariales entre trabajadores del sector público y privado", Serie Documentos de Trabajo / FCEA-IE; DT02/01.

Amarante, V., R. Arim y M. Yapor (2016), "Decomposing inequality changes in Uruguay: the role of formalization in the labor market", IZA Journal of Labor \& Development, 5(1), 13.

Attanasio, O. y C. Binelli (2010), "Mexico in the 1990s: the main cross-sectional facts", Review of Economic dynamics, 13(1), 238-264.

Becker, G. S. (1964), Human capital: A theoretical and empirical analysis, with special reference to schoolingm, National Bureau of Economic Research, Nueva York.

Binelli, C. (2016), "Wage inequality and informality: evidence from Mexico", IZA Journal of Labor \& Development, 5(1), 5.

Blinder, A. S. (1973), "Wage discrimination: reduced form and structural estimates", Journal of Human resources, 436-455.

Canelas, C. (2014), "Minimum Wage and informality in Ecuador" (No. 2014/006), Wider Working Paper.

Carrillo, P. E. (2004), "Las diferencias salariales entre el sector público y privado en el Ecuador", Cuestiones Económicas, 20(2):165-174.

Cingano, F. (2014), Trends in income inequality and its impact on economic growth.

Combes, P. P., G. Duranton y L. Gobillon (2008), "Spatial wage disparities: Sorting matters!", Journal of urban economics, 63(2), 723-742.

Dabla-Norris, M. E., M. K. Kochhar, M. N. Suphaphiphat, M. F. Ricka y E. Tsounta (2015), Causes and consequences of income inequality: A global perspective. International Monetary Fund.

De Castro, F., M. Salto y H. Steiner (2013), "The gap between public and private wages: new evidence for the EU" (No. 508), Directorate General 
Economic and Financial Affairs (DG ECFIN), European Commission.

Dell'Aringa, C., C. Lucifora y F. Origo (2007), "Public sector pay and regional competitiveness. A first look at regional public-private wage differentials in Italy". The Manchester School, 75: 445-478. doi:10.1111/j.14679957.2007.01025.x.

DiNardo, J., N. M. Fortin y T. Lemieux (1996), “Labor Market Institutions and the Distribution of Wages, 1973-1992: A Semiparametric Approach", Econometrica, 64(5), 1001-1044.

Dickey, H. (2007), "Regional earnings inequality in Great Britain: evidence from quantile regressions", Journal of Regional Science, 47(4), 775-806.

Firpo, S., N. M. Fortin y T. Lemieux (2009), “Unconditional quantile regressions", Econometrica, 77(3), 953-973.

Fortin, N., T. Lemieux y S. Firpo (2011), "Decomposition methods in economics. In Handbook of labor economics”, Elsevier, Vol. 4, pp. 1-102.

Gachet, I., D. F. Grijalva, P. Ponce y D. Rodríguez (2016), Vertical and horizontal inequality in Ecuador.

Galego, A. y J. Pereira (2014), "Decomposition of Regional Wage Differences Along the Wage Distribution in Portugal: The Importance of Covariates", Environment and Planning A, 46(10), 2514-2532. doi:10.1068/a130055p

García, I. y J. A. Molina (2002), "Inter-regional wage differentials in Spain", Applied Economics Letters, 9(4), 209-215.

Gasparini, L. y N. Lustig (2011), “The rise and fall of income inequality in Latin America" (No. 118). Documento de Trabajo.

Gasparini, L. y L. Tornarolli (2009), "Labor informality in Latin America and the Caribbean: Patterns and trends from household survey microdata", $R e$ vista Desarrollo y Sociedad, (63), 13-80.

Giordano, R., D. Depalo, M. Coutinho Pereira, B. Eugène, E. Papapetrou, J.J. Pérez, L. Reiss y M. Roter (2011), The public sector pay gap in a selection of Euro area countries.

Glaeser, E. L., H. D. Kallal, J.A. Scheinkman y A. Shleifer (1992), “Growth in cities", Journal of political economy, 100(6), 1126-1152.

Groot, S.P., H.L. de Groot y M.J. Smit (2014), "Regional wage differences in the netherlands: micro evidence on agglomeration externalities", Journal of Regional Science. 54: 503-523. doi:10.1111/jors. 12070.

Guerrero Mena, E. M. (2013), "Determinantes observables y no observables de los diferenciales salariales entre el sector público y privado en el Ecuador período 2011: un análisis empírico de descomposición de los ingresos", Tesis, Quito.

Herrera-Idárraga, P., E. López-Bazo y E. Motellón (2016), “Regional wage gaps, education and informality in an emerging country: The case of Colombia", Spatial Economic Analysis, 11(4), 432-456.

Hussmanns, R. (2004), Defining and measuring informal employment, International Labour Office, Geneva. 
Krugman, P. (1991), "Increasing returns and economic geography", Journal of political economy, 99(3), 483-499.

Lucifora, C. y D. Meurs (2006), "The public sector pay gap in France, Great Britain and Italy", Review of Income and wealth, 52(1), 43-59.

Lustig, N., L. F. Lopez-Calva y E. Ortiz-Juarez, (2012), "Declining inequality in Latin America in the 2000s: the cases of Argentina, Brazil, and Mexico", The World Bank.

Machado, J. A. y J. Mata (2005), "Counterfactual decomposition of changes in wage distributions using quantile regression", Journal of applied Econometrics, 20(4), 445-465.

Maldonado, P. C., E. Buenaño, A.L. López y F. Vásquez (2018), “Las brechas salariales público-privado e índices de bienestar: un análisis de microsimulación para Ecuador", Analitika (Revista de Analisis Estadistico/Journal of Statistical Analysis), 15(1), 39-60.

Maloney, W. F. (1999), "Does Informality Imply Segmentation in Urban Labor Markets? Evidence from Sectoral Transitions in Mexico", World Bank Economic

Review, 13 (2): 275-302.

Maloney, W. F. (2004), "Informality revisited", World development, 32(7), 1159-1178.

Maurizio, R. (2013), “Informalidad laboral y brechas salariales en América Latina. Población y trabajo en América Latina: abordajes teórico-metodológicos y tendencias empíricas recientes", 197-222.

Maurizio, R. (2014), "Labour formalization and declining inequality in Argentina and Brazil in 2000s: a dynamic approach", ILO Research Paper, (9).

Mincer, J. (1974), Schooling, Experience, and Earnings. National Bureau of Economic Research, Inc.

Motellón, E., E. López-Bazo y M. El-Attar (2011), "Regional heterogeneity in wage distributions: evidence from Spain", Journal of Regional Science, 51(3), 558-584.

Mueller, R. E. (1998), "Public-private sector wage differentials in Canada: evidence from quantile regressions", Economics Letters, 60(2), 229-235.

Muñoz, R. M. y N. Pontarollo (2016), "Cantonal Convergence in Ecuador: A Spatial Econometric Perspective”, Editorial Board, 11(1), 39.

Oaxaca, R. (1973), "Male-female wage differentials in urban labor markets", International economic review, 693-709.

Ontaneda, D. (2019), "Desigualdad Salarial y sus determinantes. El caso de Ecuador 2003-2017. Universidad de Guadalajara", Tesis de maestría, Universidad de Guadalajara, México.

Panizza, U, y C. Z. W. Qiang (2005), "Public-private wage differential and gender gap in Latin America: Spoiled bureaucrats and exploited women?", The Journal of Socio-Economics, 34(6), 810-833.

Pereira, J. y A. Galego (2014), "Inter-regional wage differentials in Portugal: 
an analysis across the wage distribution", Regional Studies, 48(9), 15291546.

Perry, G. E., O. Arias, P. Fajnzylber, W.F. Maloney, A. Mason y J. SaavedraChanduvi (2007), "Informality: Exit and exclusión", The World Bank.

Ponce, J. y R. Vos (2014), "Redistribution without structural change in Ecuador: Rising and falling income inequality in the 1990s and 2000. Falling Inequality in Latin America", Policy Changes and Lesson, Oxford, United Kingdom.

Pontarollo, N., R. Mendieta y D. Ontaneda (2019), "El crecimiento cantonal en Ecuador y el papel de la heterogeneidad espacial", Revista CEPAL.

Quintana-Romero, L., R. Correa-Quezada, M.G. Ramón-Mendieta y J. Álvarez-García (2019), "Sectoral Regional Growth and Convergence in Ecuador: An Analysis of the Intra-Distributive Dynamics of Productivity", Symmetry, 11(4), 461.

Silverman, B. W. (1986), "Density estimation for statistics and data analysis" (Vol. 26), CRC press.

Su, Z.F., X.X. Ma, W. Xiao y M.Y. Chen (2019), "Marginal Effects of Public Employment on Unconditional Distribution of Wage Income in China", The North American Journal of Economics and Finance, 101002.

Szeles, M. R. y R.M. Muñoz (2016), “Analyzing the regional economic convergence in Ecuador. Insights from parametric and nonparametric models", Romanian Journal of Economic Forecasting, 19(2), 43-65.

Zhou, Y. (2017), "Urban wage inequality and economic agglomeration", The Annals of Regional Science, 59(2), 475-494. 


\section{ANEXO}

FIgURA A.1

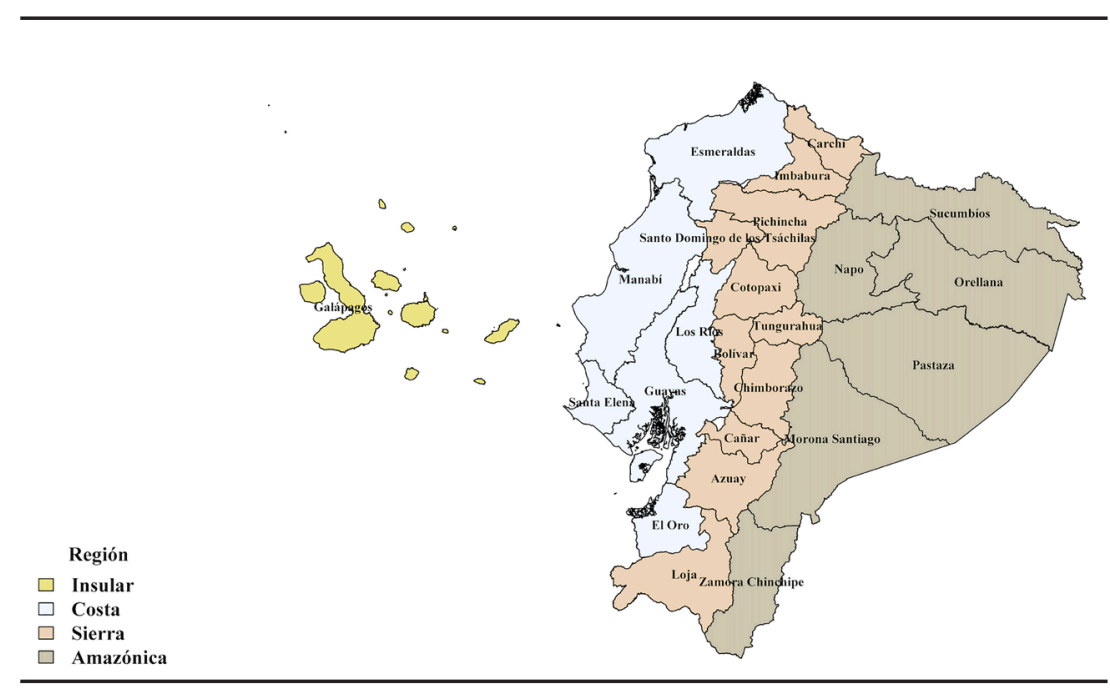

FIGURA A.2

EVOLUCIÓN DE LA DESIGUALDAD DEL INGRESO LABORAL

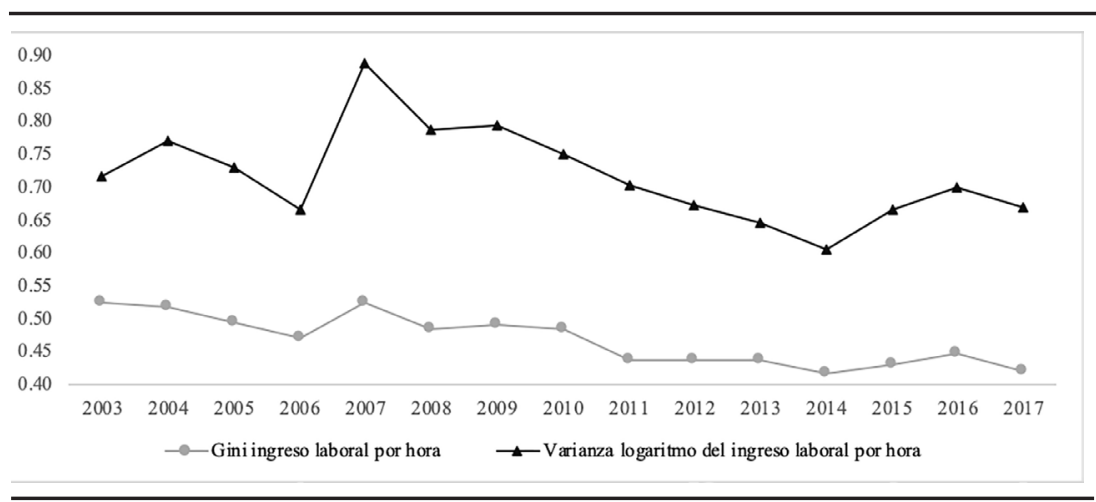

Fuente: elaboración propia con base en la Enemdu. 


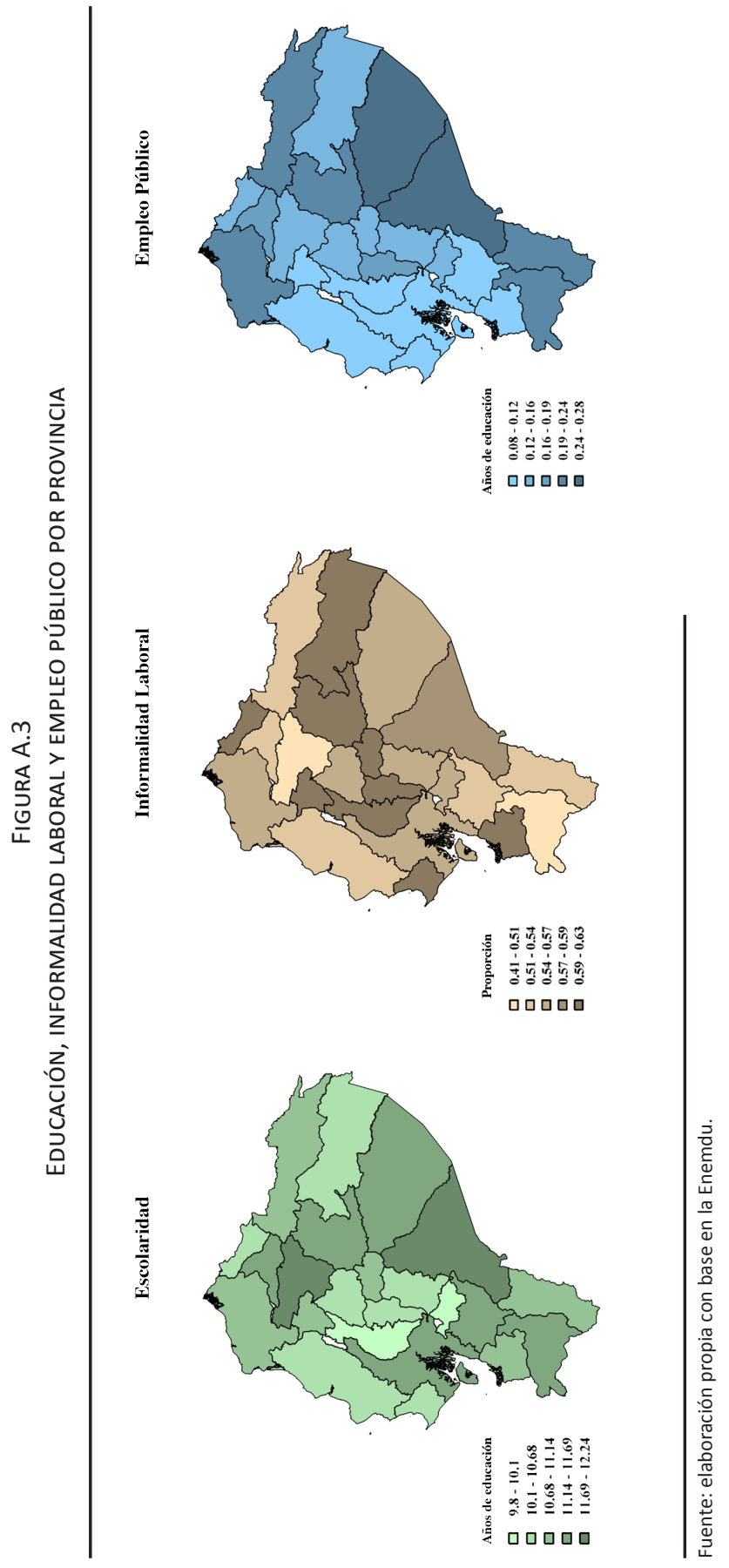


FIGURA A.4

DESCOMPOSICIÓN DE LAS BRECHAS SALARIALES POR PERCENTIL.

EFECTO CARACTERÍSTICAS

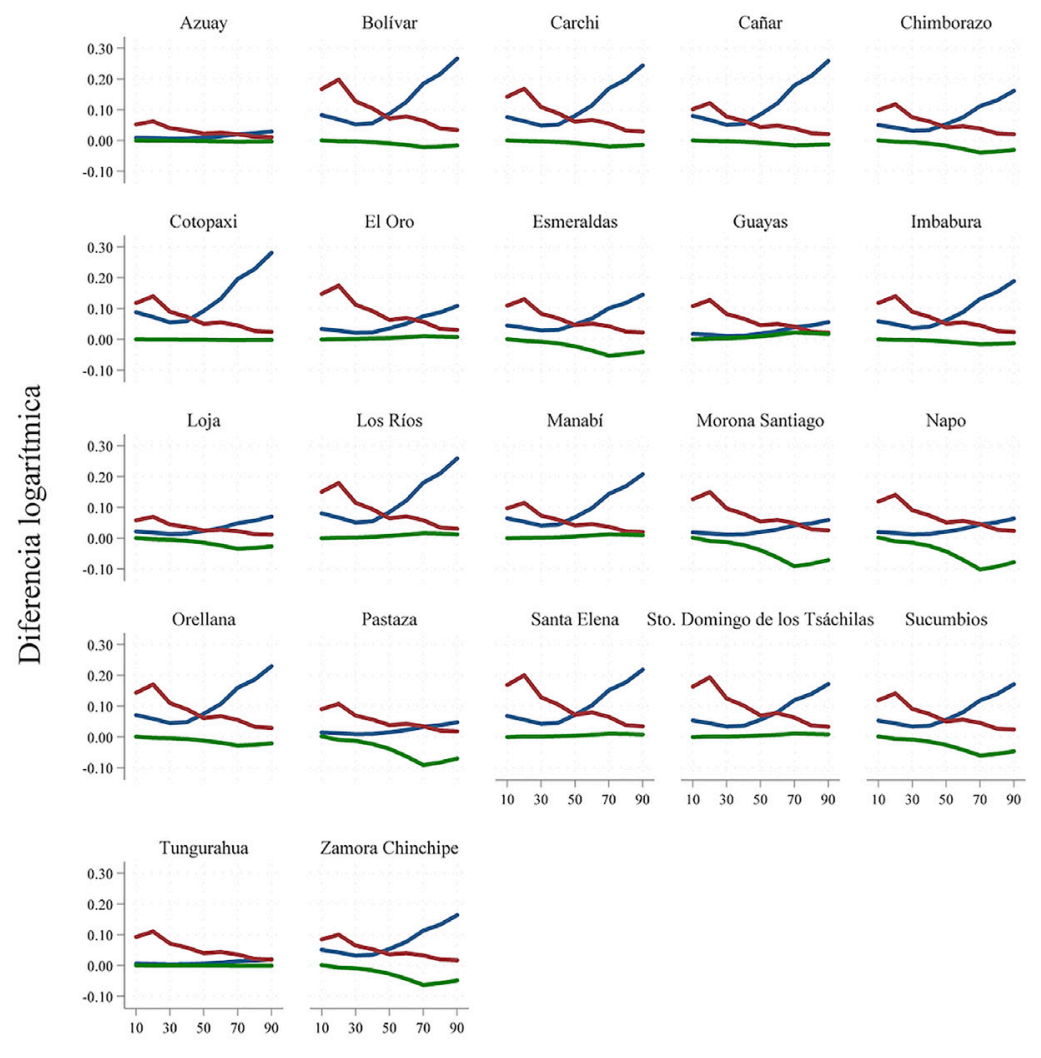

Percentiles

Educación $\quad$ Informalidad laboral $\quad$ Empleo público

Fuente: elaboración propia con base en la Enemdu. 
FIGURA A.5

DESCOMPOSICIÓN DE LAS BRECHAS SALARIALES POR PERCENTIL. EFECTO ESTRUCTURA SALARIAL

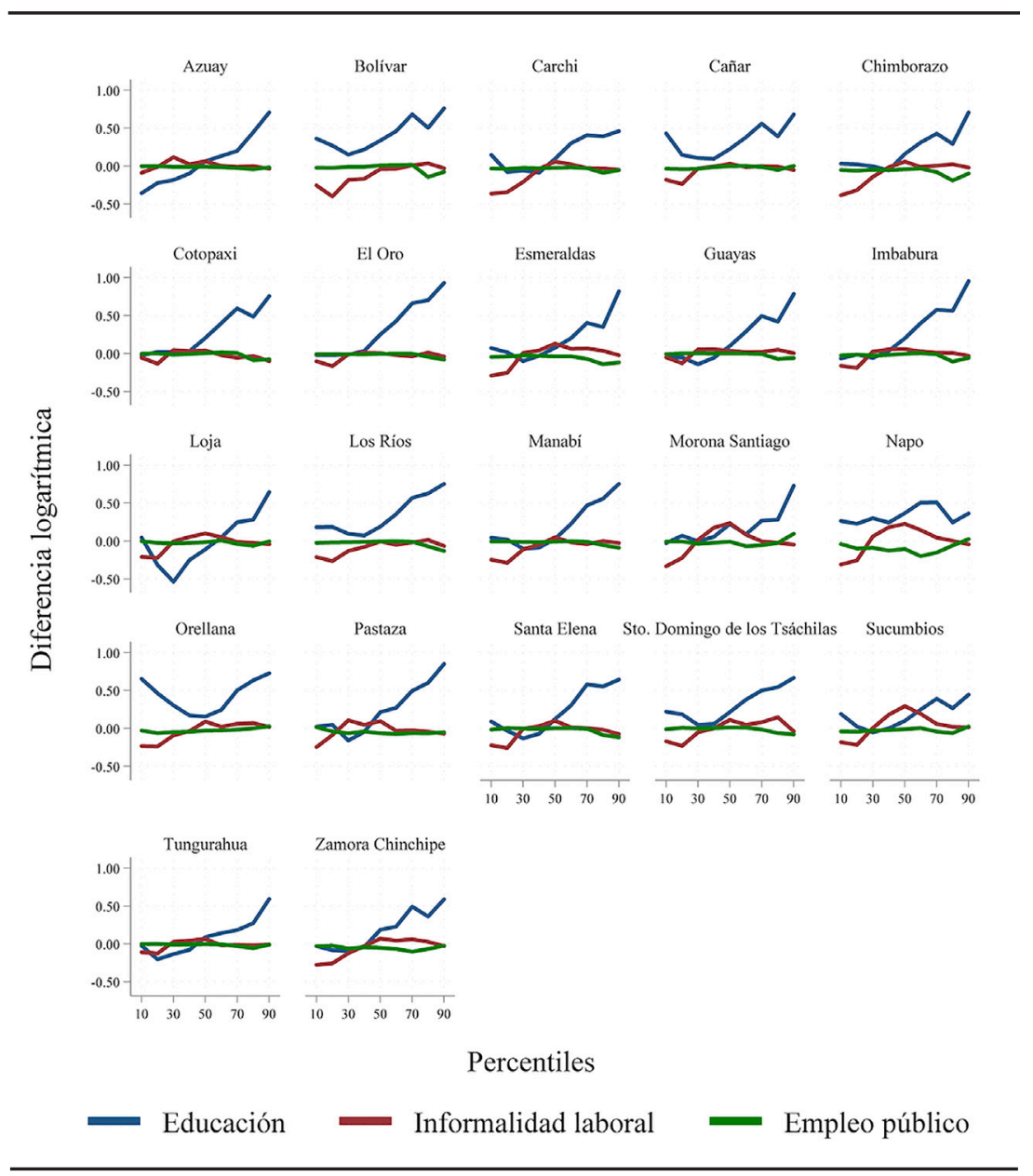

Fuente: elaboración propia con base en la Enemdu. 


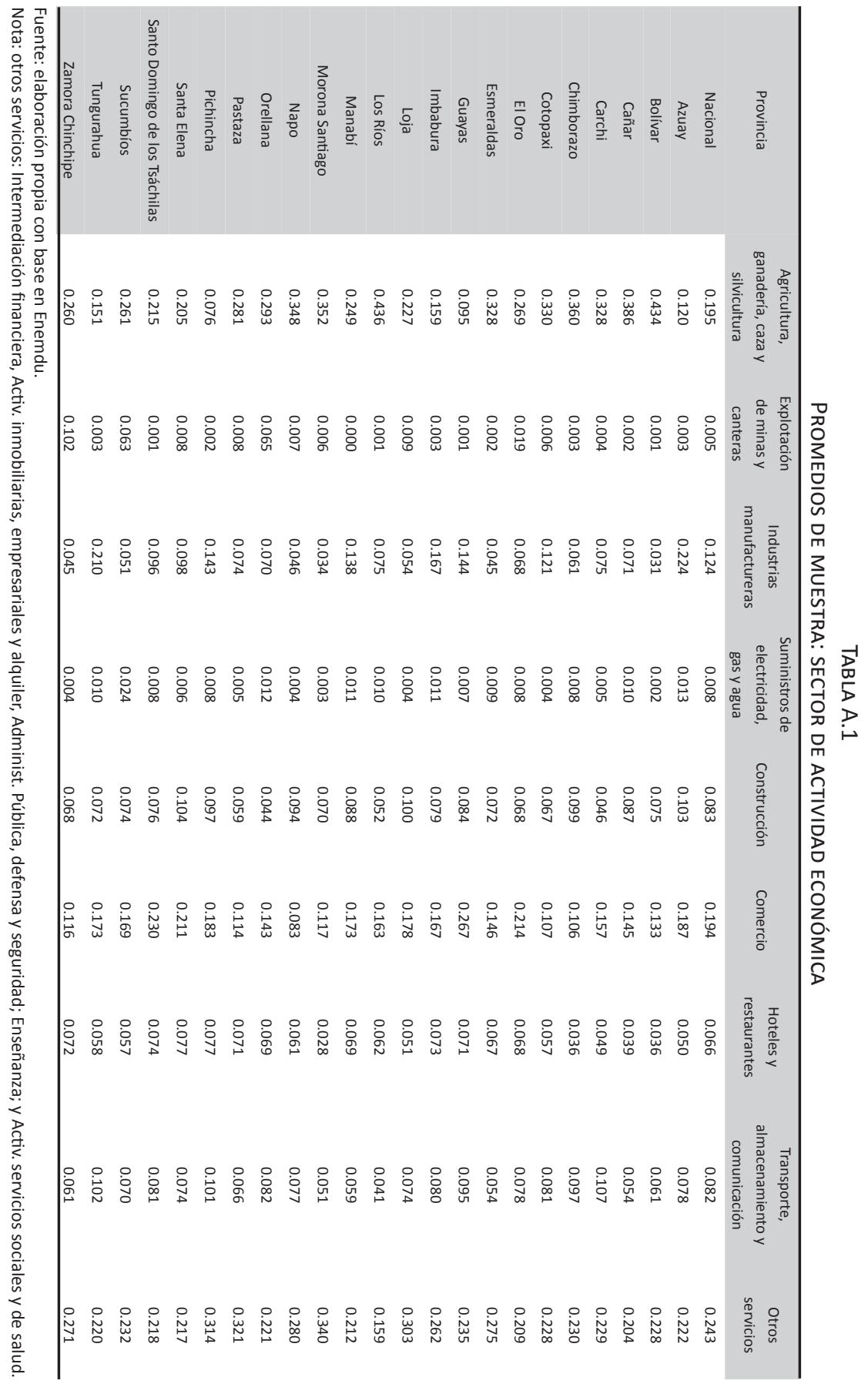


TABLA A. 2

BRECHAS SALARIALES POR PERCENTIL

\begin{tabular}{|c|c|c|c|c|c|c|}
\hline \multirow[b]{3}{*}{ Azuay } & \multicolumn{6}{|c|}{ Percentil } \\
\hline & \multicolumn{2}{|c|}{25} & \multicolumn{2}{|c|}{50} & \multicolumn{2}{|c|}{75} \\
\hline & $0.211 * * *$ & $(0.036)$ & $0.071 * * *$ & $(0.02)$ & $0.076 * *$ & $(0.033)$ \\
\hline Bolívar & $0.612^{* * *}$ & $(0.033)$ & $0.459 * * *$ & $(0.031)$ & $0.42 * * *$ & $(0.046)$ \\
\hline Cañar & $0.373^{* * *}$ & $(0.043)$ & $0.185^{* * *}$ & $(0.026)$ & $0.27^{* * *}$ & $(0.038)$ \\
\hline Carchi & $0.58 * * *$ & $(0.035)$ & $0.387 * * *$ & $(0.03)$ & $0.348^{* * *}$ & $(0.038)$ \\
\hline Cotopaxi & $0.3^{* * *}$ & $(0.032)$ & $0.19^{* * *}$ & $(0.018)$ & $0.345 * * *$ & $(0.029)$ \\
\hline Chimborazo & $0.579 * * *$ & $(0.043)$ & $0.297 * * *$ & $(0.034)$ & $0.204 * * *$ & $(0.047)$ \\
\hline El Oro & $0.237^{* * *}$ & $(0.027)$ & $0.129 * * *$ & $(0.018)$ & $0.295^{* * *}$ & $(0.028)$ \\
\hline Esmeraldas & $0.558^{* * *}$ & $(0.038)$ & $0.302 * * *$ & $(0.028)$ & $0.224 * * *$ & $(0.039)$ \\
\hline Guayas & $0.217^{* * *}$ & $(0.024)$ & $0.143^{* * *}$ & $(0.017)$ & $0.273^{* * *}$ & $(0.028)$ \\
\hline Imbabura & $0.314^{* * *}$ & $(0.03)$ & $0.188^{* * *}$ & $(0.019)$ & $0.274 * * *$ & $(0.032)$ \\
\hline Loja & $0.651 * * *$ & $(0.053)$ & $0.241^{* * *}$ & $(0.035)$ & $0.191 * * *$ & $(0.042)$ \\
\hline Los Ríos & $0.309 * * *$ & $(0.027)$ & $0.279 * * *$ & $(0.02)$ & $0.397^{* * *}$ & $(0.031)$ \\
\hline Mabí & $0.442 * * *$ & $(0.029)$ & $0.315^{* * *}$ & $(0.023)$ & $0.368^{* * *}$ & $(0.031)$ \\
\hline Morona Santiago & $0.628^{* * *}$ & $(0.054)$ & $0.189 * * *$ & $(0.039)$ & 0.049 & $(0.042)$ \\
\hline Napo & $0.795^{* * *}$ & $(0.059)$ & $0.284 * * *$ & $(0.042)$ & $0.133^{* * *}$ & $(0.041)$ \\
\hline Pastaza & $0.445^{* * *}$ & $(0.052)$ & $0.12^{* * *}$ & $(0.033)$ & $0.094 * *$ & (0.039) \\
\hline Tungurahua & $0.238 * * *$ & $(0.03)$ & $0.112^{* * *}$ & $(0.02)$ & $0.088^{* * *}$ & $(0.034)$ \\
\hline Zamora Chinchipe & $0.546^{* * *}$ & $(0.054)$ & $0.191 * * *$ & $(0.035)$ & $0.135^{* * *}$ & $(0.041)$ \\
\hline Sucumbíos & $0.67 * * *$ & $(0.049)$ & $0.303^{* * *}$ & $(0.038)$ & $0.2^{* * *}$ & $(0.042)$ \\
\hline Orellana & $0.47^{* * *}$ & $(0.043)$ & $0.302^{* * *}$ & $(0.031)$ & $0.241 * * *$ & $(0.039)$ \\
\hline Santo Domingo de los Tsáchilas & $0.282^{* * *}$ & $(0.031)$ & $0.233^{* * *}$ & $(0.023)$ & $0.339 * * *$ & $(0.036)$ \\
\hline Santa Elena & $0.43 * * *$ & $(0.032)$ & $0.282^{* * *}$ & $(0.023)$ & $0.393 * * *$ & $(0.033)$ \\
\hline
\end{tabular}

Fuente: elaboración propia con base en la Enemdu.

Errores estándar robustos entre paréntesis. ${ }^{*} p<0.1 ; * *<0.05 ; * * * p<0.001$

Nota: los errores estándar se obtienen por el método delta. 


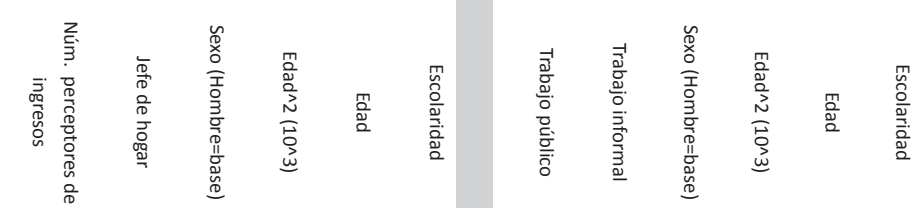

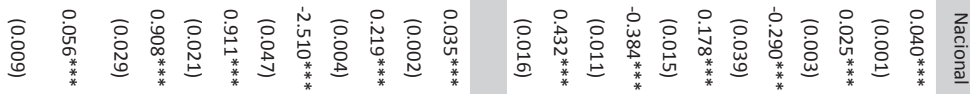

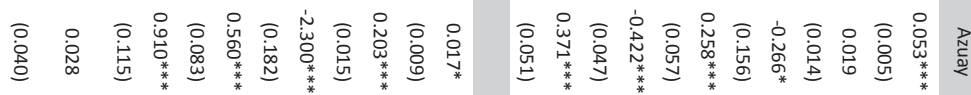

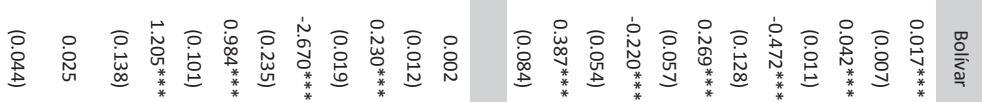

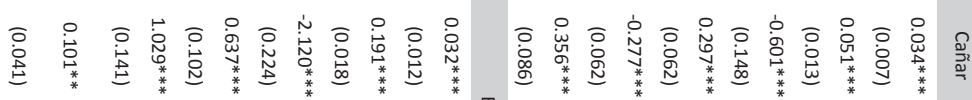

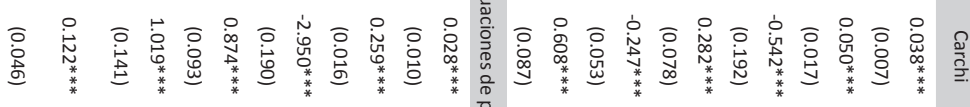

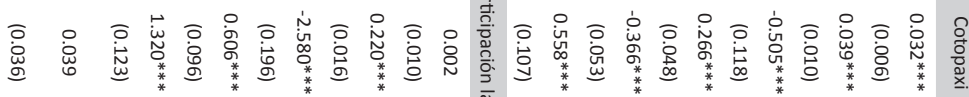

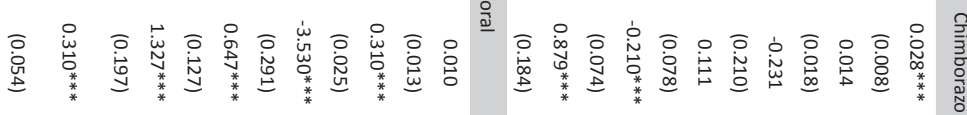

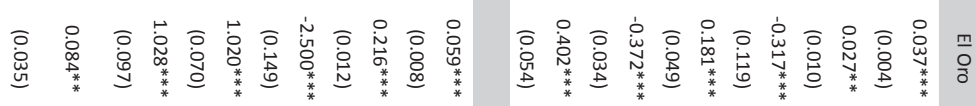

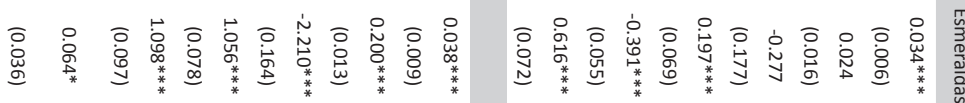

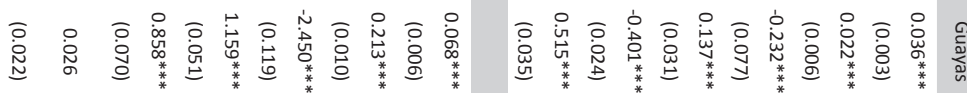

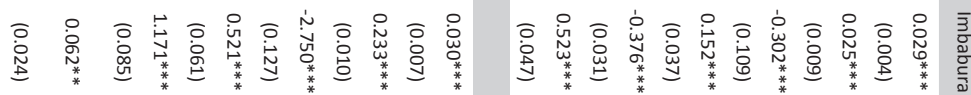

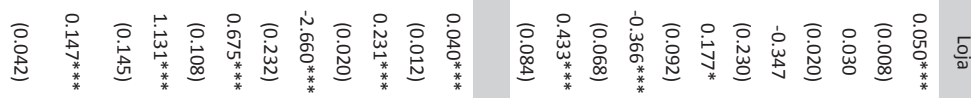




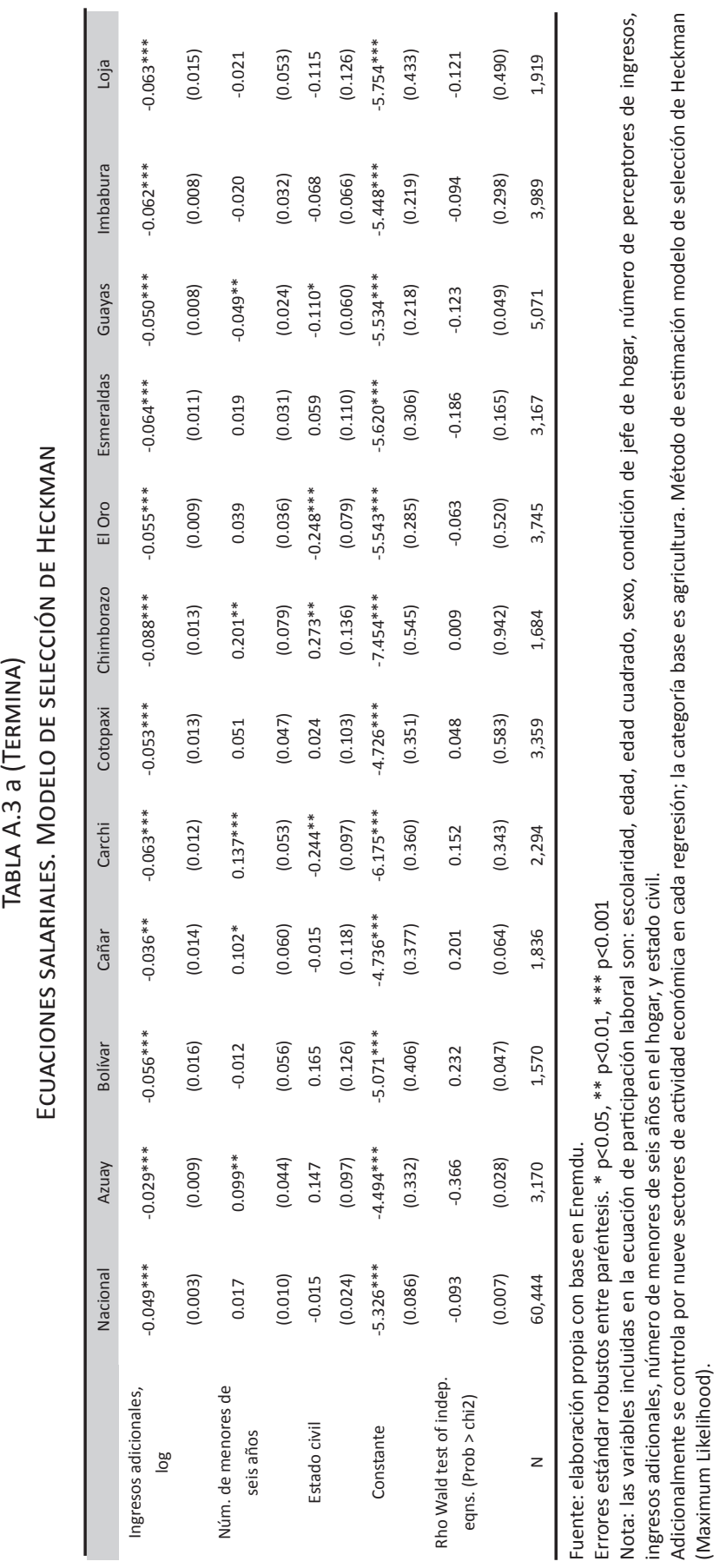




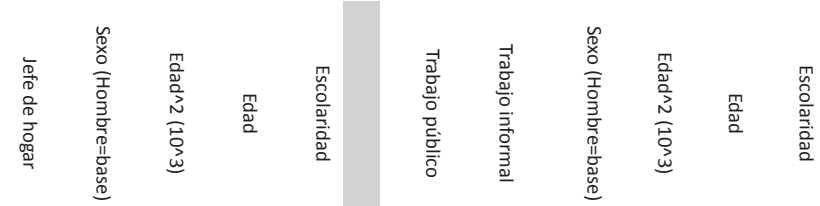

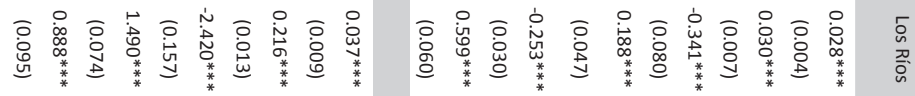

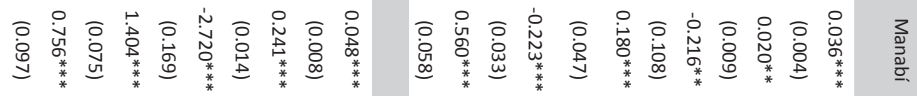

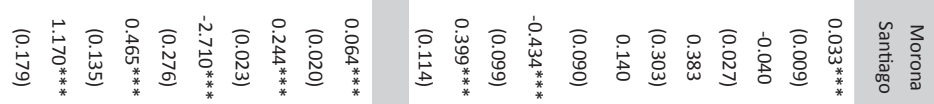

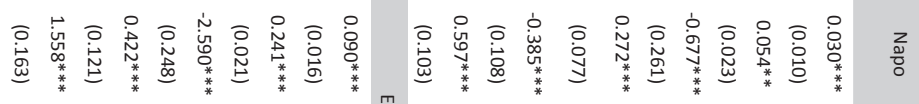

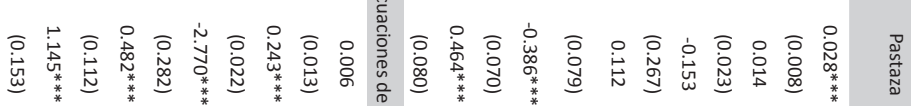

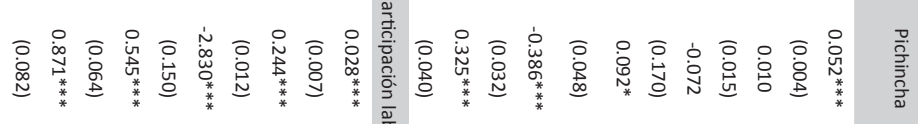

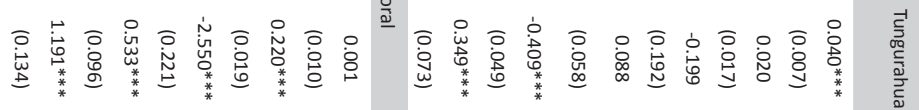

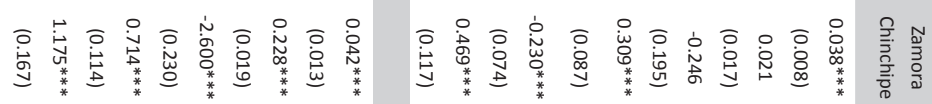

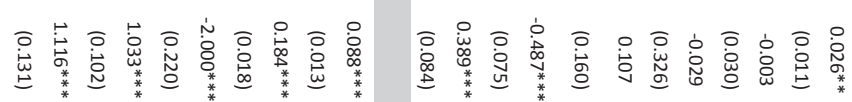

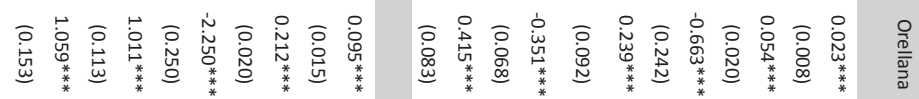

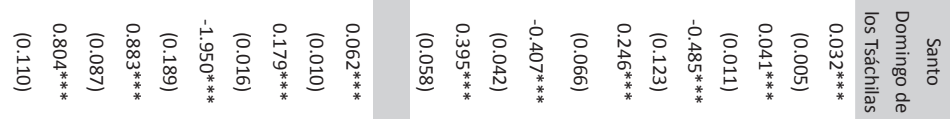

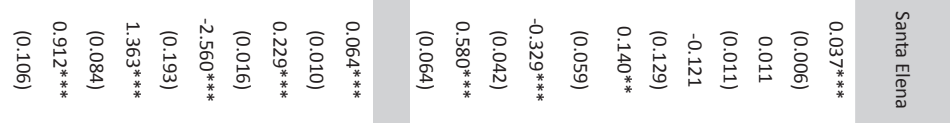




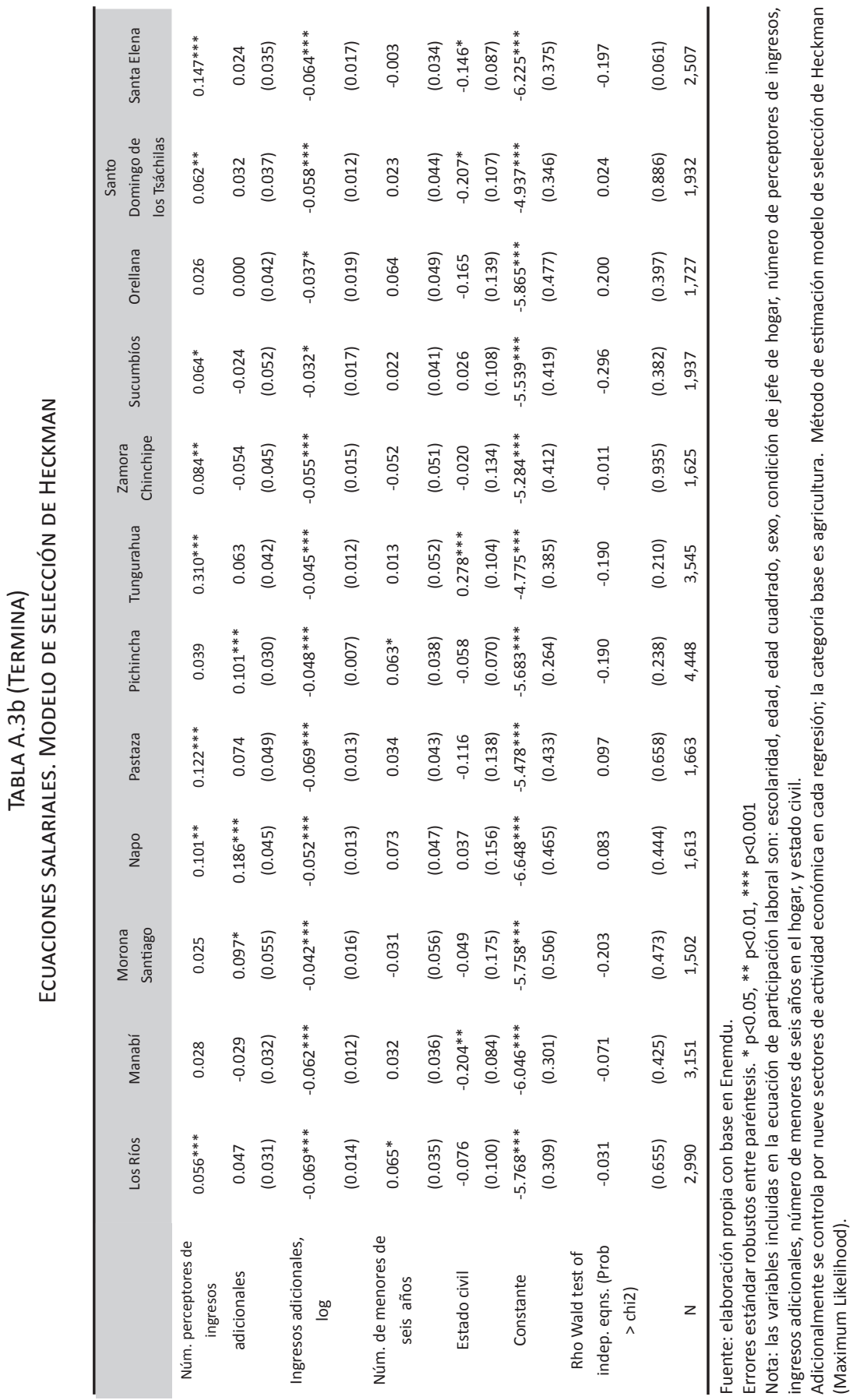

